\title{
A behavioral analysis of WOWA and SUOWA operators
}

\author{
Bonifacio Llamazares* \\ Departamento de Economía Aplicada, Instituto de Matemáticas (IMUVA), \\ and BORDA Research Unit, Universidad de Valladolid, 47011 Valladolid, Spain
}

\begin{abstract}
Weighted ordered weighted averaging (WOWA) and semiuninorm-based ordered weighted averaging (SUOWA) operators are two families of aggregation functions that simultaneously generalize weighted means and OWA operators. Both families can be obtained by using the Choquet integral with respect to normalized capacities. Therefore, they are continuous, monotonic, idempotent, compensative and homogeneous of degree 1 functions. Although both families fulfill good properties, there are situations where their behavior is quite different. The aim of this paper is to analyze both families of functions regarding some simple cases of weighting vectors, the capacities from which they are building, the weights affecting the components of each vector, and the values they return.
\end{abstract}

\section{INTRODUCTION}

Weighted means and ordered weighted averaging (OWA) operators ${ }^{1}$ are wellknown functions widely used in the aggregation processes, which are basic in many knowledge-based systems. Both families of functions are defined through weighting vectors, but their behavior is quite different: in the case of weighted means, the values are weighted according to the reliability of the information sources, whereas in the case of OWA operators, the values are weighted in accordance with their relative position.

Although weighted means and OWA operators allow to solve a wide range of problems, there are scenarios where both weightings are necessary. Some examples of these situations (in fields as diverse as robotics, vision, fuzzy logic controllers, constraint satisfaction problems, scheduling, multicriteria aggregation problems, and decision making) have been given by several authors. ${ }^{2-8}$

Different models have appeared in the literature to deal with this kind of problems. A usual approach is to consider families of functions parameterized by two weighting vectors, one for the weighted mean and the other one for the

*e-mail: boni@eco.uva.es. 
OWA-type aggregation, that generalize weighted means and OWA operators in the following sense: A weighted mean (or a OWA operator) is obtained when the other weighting vector has a "neutral" behavior; that is, it is $(1 / n, \ldots, 1 / n)$. In this framework, two of the families having better properties are the weighted OWA (WOWA) operator ${ }^{3}$ and the semiuninorm-based ordered weighted averaging (SUOWA) operator. ${ }^{8}$

WOWA and SUOWA operators can be represented by using the Choquet integral with respect to normalized capacities. In the case of the WOWA operator, the capacity is obtained by considering the image of the capacity associated with the weighted mean under a quantifier that satisfies certain properties. In turn, in the case of the SUOWA operator, its capacity is the monotonic cover of a game, which is defined by using the capacities associated with the weighted mean and the OWA operator and "assembling" these values through a semiuninorm with neutral element $1 / n$.

Because WOWA and SUOWA operators are defined through normalized capacities, they are continuous, monotonic, idempotent, compensative, and homogeneous of degree 1 functions. Since they are different families of functions, ${ }^{8}$ it seems interesting to analyze them from other points of view, to make a distinction between both families. In this sense, it is worthy of note that some results about the behavior of WOWA operators have been given by Roy ${ }^{6}$ and Llamazares. ${ }^{9}$

Given that WOWA and SUOWA operators generalize weighted means and OWA operators, we know which they are when one of the weighting vector is $(1 / n, \ldots, 1 / n)$. One of the aims of this paper is to study WOWA and SUOWA operators for some simple cases of weighting vectors. More specifically, we focus on the cases where the weighted mean is a projection or the OWA operator is an order statistic, and analyze when it is possible to recover these functions through WOWA or SUOWA operators.

The other line of research carried out in this paper analyzes some important characteristics of WOWA and SUOWA operators with regard to the corresponding ones of weighted means and OWA operators. The features studied are the capacities associated with the operators, the weights affecting the components of each vector, and the values they return.

The remainder of the paper is organized as follows. In Section 2, we recall some basic properties of aggregation functions and the concepts of semiuninorms and uninorms. Likewise, we give some interesting examples of such functions. Section 3 is dedicated to the Choquet integral, including its most interesting particular cases: weighted means, OWA operators, WOWA operators, and SUOWA operators. Section 4 is devoted to analyze WOWA and SUOWA operators for some simple cases of weighting vectors. In Section 5, we study WOWA and SUOWA operators through the capacities from which they are building, the weights affecting the components of each vector, and the values they return. Finally, some concluding remarks are provided in Section 6. 


\section{PRELIMINARIES}

Throughout the paper, we will use the following notation: $N=\{1, \ldots, n\}$; given $A \subseteq N,|A|$ denotes the cardinality of $A$; vectors are denoted in bold; $\boldsymbol{\eta}$ denotes the tuple $(1 / n, \ldots, 1 / n) \in \mathbb{R}^{n}$ and, for each $k \in N, \boldsymbol{e}_{k}$ denotes the vector with 1 in the $k$ th coordinate and 0 elsewhere. We write $\boldsymbol{x} \geq \boldsymbol{y}$ if $x_{i} \geq y_{i}$ for all $i \in N$. For a vector $\boldsymbol{x} \in \mathbb{R}^{n},[\cdot]$ and $(\cdot)$ denote permutations such that $x_{[1]} \geq \cdots \geq x_{[n]}$ and $x_{(1)} \leq \cdots \leq x_{(n)}$.

We now recall some well-known properties of aggregation functions.

Definition 1. Let $F: \mathbb{R}^{n} \longrightarrow \mathbb{R}$ be a function.

1. $F$ is symmetric if $F\left(x_{\sigma(1)}, \ldots, x_{\sigma(n)}\right)=F\left(x_{1}, \ldots, x_{n}\right)$ for all $\boldsymbol{x} \in \mathbb{R}^{n}$ and for all permutation $\sigma$ of $N$.

2. $F$ is monotonic if $\boldsymbol{x} \geq \boldsymbol{y}$ implies $F(\boldsymbol{x}) \geq F(\boldsymbol{y})$ for all $\boldsymbol{x}, \boldsymbol{y} \in \mathbb{R}^{n}$.

3. $F$ is idempotent if $F(x, \ldots, x)=x$ for all $x \in \mathbb{R}$.

4. $F$ is compensative (or internal) if $\min (\boldsymbol{x}) \leq F(\boldsymbol{x}) \leq \max (\boldsymbol{x})$ for all $\boldsymbol{x} \in$ $\mathbb{R}^{n}$.

5. F is homogeneous of degree 1 (or a ratio scale invariant) if $F(r \boldsymbol{x})=r F(\boldsymbol{x})$ for all $\boldsymbol{x} \in \mathbb{R}^{n}$ and for all $r>0$.

A class of necessary functions in the definition of SUOWA operators are semiuninorms. ${ }^{10}$ These functions are monotonic and have a neutral element in the interval $[0,1]$. They were introduced as a generalization of uninorms by dispensing with the symmetry and associativity properties. In turn, uninorms were proposed by Yager and Rybalov ${ }^{11}$ as a generalization of t-norms and tconorms.

Definition 2. Let $U:[0,1]^{2} \longrightarrow[0,1]$.

1. $U$ is a semiuninorm if it is monotonic and possesses a neutral element $e \in[0,1](U(e, x)=U(x, e)=x$ for all $x \in[0,1])$.

2. $U$ is a uninorm if it is a symmetric and associative $(U(x, U(y, z))=$ $U(U(x, y), z)$ for all $x, y, z \in[0,1])$ semiuninorm.

We denote by $\mathcal{U}^{e}$ (respectively, $\mathcal{U}_{\mathrm{i}}^{e}$ ) the set of semiuninorms (respectively, idempotent semiuninorms) with a neutral element $e \in[0,1]$. The structure of semiuninorms and idempotent semiuninorms has been studied by $\mathrm{Liu}^{10}$ (see a graphic representation in Llamazares ${ }^{8}$ ).

SUOWA operators are defined by using semiuninorms with neutral element $1 / n$. Moreover, they have to belong to the following subset: ${ }^{8}$

$$
\widetilde{\mathcal{U}}^{1 / n}=\left\{U \in \mathcal{U}^{1 / n} \mid U(1 / k, 1 / k) \leq 1 / k \text { for all } k \in N\right\} .
$$


Obviously $\mathcal{U}_{\mathrm{i}}^{1 / n} \subseteq \widetilde{\mathcal{U}}^{1 / n}$. Notice that the smallest and the largest elements of $\widetilde{\mathcal{U}}^{1 / n}$ are, respectively, the following semiuninorms:

$$
\begin{aligned}
& U_{\perp}(x, y)= \begin{cases}\max (x, y) & \text { if }(x, y) \in[1 / n, 1]^{2}, \\
0 & \text { if }(x, y) \in[0,1 / n)^{2}, \\
\min (x, y) & \text { otherwise },\end{cases} \\
& U_{\top}(x, y)= \begin{cases}1 / k & \text { if }(x, y) \in I_{k} \backslash I_{k+1}, \text { where } \\
& I_{k}=(1 / n, 1 / k]^{2} \\
\min (x, y) & \text { if }(x, y) \in[0,1 / n]^{2}, \\
\max (x, y) & \text { otherwise. }\end{cases}
\end{aligned}
$$

In the case of idempotent semiuninorms, the smallest and the largest elements of $\mathcal{U}_{\mathrm{i}}^{1 / n}$ are, respectively, the following uninorms (which were given by Yager and Rybalov $\left.^{11}\right)$ :

$$
\begin{aligned}
U_{\min }(x, y) & = \begin{cases}\max (x, y) & \text { if }(x, y) \in[1 / n, 1]^{2}, \\
\min (x, y) & \text { otherwise, }\end{cases} \\
U_{\max }(x, y) & = \begin{cases}\min (x, y) & \text { if }(x, y) \in[0,1 / n]^{2}, \\
\max (x, y) & \text { otherwise. }\end{cases}
\end{aligned}
$$

In addition to the previous ones, several procedures to construct semiuninorms have been introduced by Llamazares. ${ }^{12}$ One of them, which is based on ordinal sums of aggregation operators, allows us to get continuous semiuninorms. One of the most relevant continuous semiuninorm obtained is the following:

$$
U_{T_{\mathbf{L}}}(x, y)= \begin{cases}\max (x, y) & \text { if }(x, y) \in[1 / n, 1]^{2}, \\ \max (x+y-1 / n, 0) & \text { otherwise }\end{cases}
$$

The plots of all these semiuninorms for the case $n=4$ can be found in Llamazares. ${ }^{13}$

\section{CHOQUET INTEGRAL}

The Choquet integral ${ }^{14}$ was introduced in 1953 , and since then it has had a wide variety of applications due to their simplicity and versatility. ${ }^{15,16}$ The notion of the Choquet integral is based on that of capacity. ${ }^{14,17}$ The concept of capacity, which was also introduced by Sugeno ${ }^{18}$ as a fuzzy measure, resembles that of probability measure, but in the definition of the former additivity is replaced by monotonicity. A game is then a generalization of a capacity where the monotonicity is no longer required.

\section{DeFinition 3.}

1. A game $v$ on $N$ is a set function, $v: 2^{N} \longrightarrow \mathbb{R}$ satisfying $v(\varnothing)=0$. 
2. A capacity (or fuzzy measure) $\mu$ on $N$ is a game on $N$ satisfying $\mu(A) \leq$ $\mu(B)$ whenever $A \subseteq B$. In particular, it follows that $\mu: 2^{N} \longrightarrow[0, \infty) . A$ capacity $\mu$ is said to be normalized if $\mu(N)=1$.

A straightforward way to get a capacity from a game is to consider the monotonic cover of the game. ${ }^{19,20}$

Definition 4. Let $v$ be a game on $N$. The monotonic cover of $v$ is the set function $\hat{v}$ given by

$$
\hat{v}(A)=\max _{B \subseteq A} v(B) .
$$

Some basic properties of $\hat{v}$ are given in the sequel.

Remark 1 . Let $v$ be a game on $N$. Then:

1. $\hat{v}$ is a capacity.

2. If $v$ is a capacity, then $\hat{v}=v$.

3. If $v(A) \leq 1$ for all $A \subseteq N$ and $v(N)=1$, then $\hat{v}$ is a normalized capacity.

The Choquet integral is usually defined as a functional, ${ }^{14,17,21}$ but in the discrete case and once the capacity has been chosen it can be seen as an aggregation function over $\mathbb{R}^{n} .{ }^{22}$ This is the approach taken in this paper. Moreover, by analogy with the original definition of OWA operators, we represent it by using a decreasing sequence of values: ${ }^{8,23}$

Definition 5. Let $\mu$ be a capacity on $N$. The Choquet integral with respect to $\mu$ is the function $\mathcal{C}_{\mu}: \mathbb{R}^{n} \longrightarrow \mathbb{R}$ given by

$$
\mathcal{C}_{\mu}(\boldsymbol{x})=\sum_{i=1}^{n} \mu\left(A_{[i]}\right)\left(x_{[i]}-x_{[i+1]}\right),
$$

where $A_{[i]}=\{[1], \ldots,[i]\}$, and we use the convention $x_{[n+1]}=0$.

From this definition, it is straightforward to show explicitly the weights of the components $x_{[i]}$ by representing the Choquet integral as follows:

$$
\mathcal{C}_{\mu}(\boldsymbol{x})=\sum_{i=1}^{n}\left(\mu\left(A_{[i]}\right)-\mu\left(A_{[i-1]}\right)\right) x_{[i]},
$$

where we use the convention $A_{[0]}=\varnothing$. It is worth noting that the Choquet integral possesses properties ${ }^{22}$ which are useful in certain information aggregation contexts. $^{\mathrm{a}}$

Remark 2. Let $\mu$ be a capacity on $N$. Then $\mathcal{C}_{\mu}$ is continuous, monotonic and homogeneous of degree 1 . Moreover, it is idempotent and compensative when $\mu$ is a normalized capacity.

\footnotetext{
${ }^{a}$ Although, for instance, monotonicity is a very restrictive property in certain areas. ${ }^{24}$
} 
Remark 3. Let $\mu_{1}$ and $\mu_{2}$ be two capacities on $N$. Then $\mu_{1} \leq \mu_{2}{ }^{\mathrm{b}}$ if and only if $\mathcal{C}_{\mu_{1}} \leq \mathcal{C}_{\mu_{2}}$.

In the following subsections, we recollect the most important particular cases of the Choquet integral: weighted means, OWA operators, WOWA operators, and SUOWA operators.

\subsection{Weighted means and OWA operators}

Weighted means and OWA operators ${ }^{1}$ are well-known functions in the theory of aggregation operators (see Emrouznejad and Marra ${ }^{25}$ in the case of OWA operators). Both classes of functions are defined in terms of weight distributions that add up to 1 .

Definition 6. A vector $\boldsymbol{q} \in[0,1]^{n}$ is a weighting vector if $\sum_{i=1}^{n} q_{i}=1$.

DeFinition 7. Let $\boldsymbol{p}$ be a weighting vector. The weighted mean associated with $\boldsymbol{p}$ is the function $M_{\boldsymbol{p}}: \mathbb{R}^{n} \longrightarrow \mathbb{R}$ given by

$$
M_{\boldsymbol{p}}(\boldsymbol{x})=\sum_{i=1}^{n} p_{i} x_{i} .
$$

DeFINITION 8. Let $\boldsymbol{w}$ be a weighting vector. The OWA operator associated with $\boldsymbol{w}$ is the function $O_{\boldsymbol{w}}: \mathbb{R}^{n} \longrightarrow \mathbb{R}$ given by

$$
O_{\boldsymbol{w}}(\boldsymbol{x})=\sum_{i=1}^{n} w_{i} x_{[i]} .
$$

It is well known that weighted means and OWA operators are Choquet integrals with respect to normalized capacities. ${ }^{8,26-28}$

\section{Remark 4.}

1. If $\boldsymbol{p}$ is a weighting vector, then the weighted mean $M_{\boldsymbol{p}}$ is the Choquet integral with respect to the normalized capacity $\mu_{\boldsymbol{p}}(A)=\sum_{i \in A} p_{i}$.

2. If $\boldsymbol{w}$ is a weighting vector, then the OWA operator $O_{\boldsymbol{w}}$ is the Choquet integral with respect to the normalized capacity $\mu_{|\boldsymbol{w}|}(A)=\sum_{i=1}^{|A|} w_{i}$.

So, according to Remark 2, weighted means and OWA operators are continuous, monotonic, idempotent, compensative, and homogeneous of degree 1. Moreover, in the case of OWA operators, given that the values of the variables are previously ordered in a decreasing way, they are also symmetric.

\footnotetext{
${ }^{\mathrm{b}}$ The symbol $\leq$ in the middle of two functions represents the usual order between them; in this case, $\mu_{1} \leq \mu_{2}$ if $\mu_{1}(A) \leq \mu_{2}(A)$ for all $A \subseteq N$.
} 


\subsection{WOWA operators}

WOWA operators ${ }^{3}$ were introduced to consider situations where both the importance of information sources and the importance of values had to be taken into account. Initially, they were defined by using monotonic functions that interpolates the points $\left(i / n, \sum_{j=1}^{i} w_{j}\right)$ together with the point $(0,0)$. But, given that quantifiers satisfy these properties, Torra and Godo ${ }^{29}$ suggested an alternative definition by using these functions. The relationship between quantifiers and the weighting vectors $\boldsymbol{w}$ was given by Yager. ${ }^{30}$.

Definition 9. A function $Q:[0,1] \longrightarrow[0,1]$ is a quantifier if it satisfies the following properties:

1. $Q(0)=0, Q(1)=1$.

2. $x>y \Rightarrow Q(x) \geq Q(y)$; i.e., it is a monotonic function.

Given a quantifier $Q$, we can obtain a weighting vector $\boldsymbol{w}$ by means of the following expression:

$$
w_{i}=Q\left(\frac{i}{n}\right)-Q\left(\frac{i-1}{n}\right), \quad i=1, \ldots, n .
$$

In this case, we will say that the quantifier $Q$ generates the weighting vector $\boldsymbol{w}$. Notice that $Q$ interpolates the points $\left(i / n, \sum_{j=1}^{i} w_{j}\right)$ together with the point $(0,0)$.

DEFINITION 10. Let $\boldsymbol{p}$ and $\boldsymbol{w}$ be two weighting vectors and let $Q$ be a quantifier generating the weighting vector $\boldsymbol{w}$. The WOWA operator associated with $\boldsymbol{p}, \boldsymbol{w}$ and $Q$ is the function $W_{\boldsymbol{p}, \boldsymbol{w}}^{Q}: \mathbb{R}^{n} \longrightarrow \mathbb{R}$ given by

$$
W_{\boldsymbol{p}, \boldsymbol{w}}^{Q}(\boldsymbol{x})=\sum_{i=1}^{n} q_{i} x_{[i]},
$$

where the weight $q_{i}$ is defined as

$$
q_{i}=Q\left(\sum_{j=1}^{i} p_{[j]}\right)-Q\left(\sum_{j=1}^{i-1} p_{[j]}\right) .
$$

It is worth noting that, in order to generalize the weighted mean $M_{\boldsymbol{p}}$, it is necessary that the quantifier $Q$ be the identity when $\boldsymbol{w}=\boldsymbol{\eta}$. Likewise, notice that WOWA operators are a specific case of the Choquet integral. ${ }^{23}$

Remark 5. If $\boldsymbol{p}$ and $\boldsymbol{w}$ are two weighting vectors and $Q$ is a quantifier generating the weighting vector $\boldsymbol{w}$, then $W_{\boldsymbol{p}, \boldsymbol{w}}^{Q}$ is the Choquet integral with respect to the normalized capacity $\mu_{\boldsymbol{p}, \boldsymbol{w}}^{Q}(A)=Q\left(\mu_{\boldsymbol{p}}(A)\right)=Q\left(\sum_{i \in A} p_{i}\right)$. Moreover, according to expression (1), the WOWA operator can also be written as

$$
W_{\boldsymbol{p}, \boldsymbol{w}}^{Q}(\boldsymbol{x})=\sum_{i=1}^{n} Q\left(\sum_{j=1}^{i} p_{[j]}\right)\left(x_{[i]}-x_{[i+1]}\right) .
$$


WOWA operators generalize weighted means and OWA operators in the sense that $W_{\boldsymbol{p}, \boldsymbol{\eta}}^{Q}=M_{\boldsymbol{p}}$ and $W_{\boldsymbol{\eta}, \boldsymbol{w}}^{Q}=O_{\boldsymbol{w}}$. Moreover, according to Remark 2, they are continuous, monotonic, idempotent, compensative, and homogeneous of degree $1 .{ }^{3}$

\subsection{SUOWA operators}

SUOWA operators ${ }^{8}$ were introduced as an alternative to WOWA operators. These functions are also a particular case of the Choquet integral where their capacities are the monotonic cover of specific games. These games are defined by using semiuninorms with neutral element $1 / n$ and the values of the capacities associated with the weighted means and the OWA operators. To be specific, the games from which SUOWA operators are built are defined as follows.

DeFINITION 11. Let $\boldsymbol{p}$ and $\boldsymbol{w}$ be two weighting vectors and let $U \in \widetilde{\mathcal{U}}^{1 / n}$.

1. The game associated with $\boldsymbol{p}, \boldsymbol{w}$ and $U$ is the set function $v_{\boldsymbol{p}, \boldsymbol{w}}^{U}: 2^{N} \longrightarrow \mathbb{R}$ defined by

$$
v_{\boldsymbol{p}, \boldsymbol{w}}^{U}(A)=|A| U\left(\frac{\mu_{\boldsymbol{p}}(A)}{|A|}, \frac{\mu_{|\boldsymbol{w}|}(A)}{|A|}\right)
$$

if $A \neq \varnothing$, and $v_{\boldsymbol{p}, \boldsymbol{w}}^{U}(\varnothing)=0$.

2. $\hat{v}_{\boldsymbol{p}, \boldsymbol{w}}^{U}$, the monotonic cover of the game $v_{\boldsymbol{p}, \boldsymbol{w}}^{U}$, will be called the capacity associated with $\boldsymbol{p}, \boldsymbol{w}$ and $U$.

Notice that $v_{\boldsymbol{p}, \boldsymbol{w}}^{U}(A) \leq 1$ for all $A \subseteq N$ and $v_{\boldsymbol{p}, \boldsymbol{w}}^{U}(N)=1$. Therefore, according to the third item of Remark $1, \hat{v}_{\boldsymbol{p}, \boldsymbol{w}}^{U}$ is always a normalized capacity.

DeFinition 12. Let $\boldsymbol{p}$ and $\boldsymbol{w}$ be two weighting vectors and let $U \in \widetilde{\mathcal{U}}^{1 / n}$. The SUOWA operator associated with $\boldsymbol{p}, \boldsymbol{w}$ and $U$ is the function $S_{\boldsymbol{p}, \boldsymbol{w}}^{U}: \mathbb{R}^{n} \longrightarrow \mathbb{R}$ given by

$$
S_{\boldsymbol{p}, \boldsymbol{w}}^{U}(\boldsymbol{x})=\sum_{i=1}^{n} s_{i} x_{[i]},
$$

where $s_{i}=\hat{v}_{\boldsymbol{p}, \boldsymbol{w}}^{U}\left(A_{[i]}\right)-\hat{v}_{\boldsymbol{p}, \boldsymbol{w}}^{U}\left(A_{[i-1]}\right)$ for all $i \in N, \hat{v}_{\boldsymbol{p}, \boldsymbol{w}}^{U}$ is the capacity associated with $\boldsymbol{p}, \boldsymbol{w}$ and $U$, and $A_{[i]}=\{[1], \ldots,[i]\}$ (with the convention that $A_{[0]}=\varnothing$ ).

According to expression (1), the SUOWA operator associated with $\boldsymbol{p}, \boldsymbol{w}$ and $U$ can also be written as

$$
S_{\boldsymbol{p}, \boldsymbol{w}}^{U}(\boldsymbol{x})=\sum_{i=1}^{n} \hat{v}_{\boldsymbol{p}, \boldsymbol{w}}^{U}\left(A_{[i]}\right)\left(x_{[i]}-x_{[i+1]}\right) .
$$

By the choice of $\hat{v}_{\boldsymbol{p}, \boldsymbol{w}}^{U}$ we have $S_{\boldsymbol{p}, \boldsymbol{\eta}}^{U}=M_{\boldsymbol{p}}$ and $S_{\boldsymbol{\eta}, \boldsymbol{w}}^{U}=O_{\boldsymbol{w}}$ for any $U \in$ $\widetilde{\mathcal{U}}^{1 / n}$. Moreover, by Remark 2 and given that $\hat{v}_{\boldsymbol{p}, \boldsymbol{w}}^{U}$ is a normalized capacity, SUOWA operators are continuous, monotonic, idempotent, compensative, and homogeneous of degree 1 . 
It is worthy of note that SUOWA operators preserve the order of the corresponding semiuninorms. As an immediate consequence of this fact, we know the bounds of SUOWA operators when we consider generic semiuninorms or idempotent semiuninorms.

Proposition 1 (Llamazares $^{8}$ ). Let $\boldsymbol{p}$ and $\boldsymbol{w}$ be two weighting vectors. Then the following holds:

1. If $U_{1}, U_{2} \in \widetilde{\mathcal{U}}^{1 / n}$ and $U_{1} \leq U_{2}$, then $S_{\boldsymbol{p}, \boldsymbol{w}}^{U_{1}} \leq S_{\boldsymbol{p}, \boldsymbol{w}}^{U_{2}}$.

2. If $U \in \widetilde{\mathcal{U}}^{1 / n}$, then $S_{\boldsymbol{p}, \boldsymbol{w}}^{U_{\perp}} \leq S_{\boldsymbol{p}, \boldsymbol{w}}^{U} \leq S_{\boldsymbol{p}, \boldsymbol{w}}^{U_{\top}}$.

3. If $U \in \mathcal{U}_{\mathrm{i}}^{1 / n}$, then $S_{\boldsymbol{p}, \boldsymbol{w}}^{U_{\min }} \leq S_{\boldsymbol{p}, \boldsymbol{w}}^{U} \leq S_{\boldsymbol{p}, \boldsymbol{w}}^{U_{\max }}$.

\section{SPECIFIC CASES OF WEIGHTING VECTORS}

WOWA and SUOWA operators have been built for generalizing simultaneously weighted means and OWA operators. So, we know their behavior when $\boldsymbol{p}=\boldsymbol{\eta}$ or when $\boldsymbol{w}=\boldsymbol{\eta}$ (recall that $\boldsymbol{\eta}=(1 / n, \ldots, 1 / n))$. The aim of this section is to analyze both families when we consider simple cases of the weighting vectors $\boldsymbol{p}$ and $\boldsymbol{w}$. More specifically, we focus on the weighting vectors $\boldsymbol{p}=\boldsymbol{e}_{k}$ and $\boldsymbol{w}=\boldsymbol{e}_{l}$, where $k, l \in N$, and analyze when WOWA and SUOWA operators allow us to recover the projection $M_{\boldsymbol{e}_{k}}$ and the order statistic $O_{\boldsymbol{e}_{l}}$.

In the sequel, we show explicitly the capacities of weighted means and OWA operators when the weighting vectors are $\boldsymbol{p}=\boldsymbol{e}_{k}$ and $\boldsymbol{w}=\boldsymbol{e}_{l}$, respectively. Notice that when $\boldsymbol{p}=\boldsymbol{e}_{k}$ the capacity $\mu_{\boldsymbol{e}_{k}}$ is known as the Dirac measure centered on $k$ and when $\boldsymbol{w}=\boldsymbol{e}_{l}$ the capacity $\mu_{\left|\boldsymbol{e}_{l}\right|}$ is known as the threshold measure $\tau_{l}{ }^{22}$

Remark 6 . Let $k, l \in N$. The following holds:

1. If $\boldsymbol{p}=\boldsymbol{e}_{k}$, then

$$
\mu_{\boldsymbol{e}_{k}}(A)=\sum_{i \in A} p_{i}= \begin{cases}0 & \text { if } k \notin A \\ 1 & \text { if } k \in A\end{cases}
$$

2. If $\boldsymbol{w}=\boldsymbol{e}_{l}$, then

$$
\mu_{\left|e_{l}\right|}(A)=\sum_{i=1}^{|A|} w_{i}= \begin{cases}0 & \text { if }|A|<l \\ 1 & \text { if }|A| \geq l\end{cases}
$$

\subsection{WOWA operators}

We first consider the weighting vector $\boldsymbol{p}=\boldsymbol{e}_{k}$. In this case, it is known that given any weighting vector $\boldsymbol{w}$ and any quantifier $Q$ generating the weighting vector $\boldsymbol{w}$, the WOWA operator associated with $\boldsymbol{e}_{k}, \boldsymbol{w}$ and $Q$ coincides with the projection $M_{e_{k}}$. 
Proposition 2 (Torra $\left.{ }^{3}\right)$. If $k \in N$, then for any weighting vector $\boldsymbol{w}$ and for any quantifier $Q$ generating the weighting vector $\boldsymbol{w}$ we have $W_{\boldsymbol{e}_{k}, \boldsymbol{w}}^{Q}=M_{\boldsymbol{e}_{k}}$.

We now examine the case $\boldsymbol{w}=\boldsymbol{e}_{l}$. In the sequel, we give a necessary and sufficient condition for the existence of a quantifier $Q$ generating the weighting vector $\boldsymbol{e}_{l}$ so that the WOWA operator associated with $\boldsymbol{p}, \boldsymbol{e}_{l}$, and $Q$ coincides with the order statistic $O_{\boldsymbol{e}_{l}}$. The condition is that the sum of $l-1$ largest components of $\boldsymbol{p}$ is less than the sum of $l$ smallest components of $\boldsymbol{p}$. For the sake of simplicity, we can suppose, without loss of generality, that the components of the weighting vector $\boldsymbol{p}$ are ordered in a decreasing way.

Proposition 3. Let $l \in N$ and let $\boldsymbol{p}$ be a weighting vector such that $p_{1} \geq p_{2} \geq$ $\cdots \geq p_{n}$. The following conditions are equivalent:

1. There exists a quantifier $Q$ generating the weighting vector $\boldsymbol{e}_{l}$ such that $W_{\boldsymbol{p}, \boldsymbol{e}_{l}}^{Q}=O_{\boldsymbol{e}_{l}}$.

2. $p_{1}+\cdots+p_{l-1}<p_{n-(l-1)}+\cdots+p_{n}$.

Proof.

$(1) \Rightarrow(2)$ : Consider $A=\{1,2, \ldots, l-1\}$ and $B=\{n-(l-1), n-(l-$ $2), \ldots, n\}$. Then $Q\left(p_{1}+\cdots+p_{l-1}\right)=\mu_{\boldsymbol{p}, \boldsymbol{e}_{l}}^{Q}(A)=\mu_{\left|\boldsymbol{e}_{l}\right|}(A)=0$ and $Q\left(p_{n-(l-1)}+\right.$ $\left.\cdots+p_{n}\right)=\mu_{\boldsymbol{p}, \boldsymbol{e}_{l}}^{Q}(B)=\mu_{\left|\boldsymbol{e}_{l}\right|}(B)=1$. Since $Q$ is a monotonic function, we have $p_{1}+\cdots+p_{l-1}<p_{n-(l-1)}+\cdots+p_{n}$.

$(2) \Rightarrow(1)$ : Let $p^{\prime}$ be located between $p_{1}+\cdots+p_{l-1}$ and $p_{n-(l-1)}+\cdots+p_{n}$; that is, $p_{1}+\cdots+p_{l-1}<p^{\prime}<p_{n-(l-1)}+\cdots+p_{n}$, and let $Q$ be the quantifier defined by

$$
Q(x)= \begin{cases}0 & \text { if } x \leq p^{\prime} \\ 1 & \text { if } x>p^{\prime}\end{cases}
$$

Since $p_{1} \geq p_{2} \geq \cdots \geq p_{n}$ and $p_{1}+\cdots+p_{n}=1$, we get $p_{1}+\cdots+p_{l-1} \geq(l-1) / n$ and $p_{n-(l-1)}+\cdots+p_{n} \leq l / n$. Therefore, $(l-1) / n<p^{\prime}$ and $l / n>p^{\prime}$ and, consequently, $Q((l-1) / n)=0$ and $Q(l / n)=1$; that is, $Q$ generates the weighting vector $\boldsymbol{e}_{l}$. Now we are going to see that $\mu_{\boldsymbol{p}, \boldsymbol{e}_{l}}^{Q}=\mu_{\left|\boldsymbol{e}_{l}\right|}$. Given $A \subseteq N$, we distinguish two cases:

1. If $|A|<l$, then $\mu_{\boldsymbol{p}, \boldsymbol{e}_{l}}^{Q}(A)=Q\left(\sum_{i \in A} p_{i}\right) \leq Q\left(p_{1}+\cdots+p_{l-1}\right)=0$; that is, $\mu_{\boldsymbol{p}, \boldsymbol{e}_{l}}^{Q}(A)=0$.

2. If $|A| \geq l$, then $\mu_{\boldsymbol{p}, \boldsymbol{e}_{l}}^{Q}(A)=Q\left(\sum_{i \in A} p_{i}\right) \geq Q\left(p_{n-(l-1)}+\cdots+p_{n}\right)=1$; that is, $\mu_{\boldsymbol{p}, \boldsymbol{e}_{l}}^{Q}(A)=1$.

So, according to the second item of Remark $6, \mu_{\boldsymbol{p}, \boldsymbol{e}_{l}}^{Q}=\mu_{\left|\boldsymbol{e}_{l}\right|}$.

Some specific cases are given in the sequel.

Corollary 1. Let $\boldsymbol{p}$ be a weighting vector. The following holds:

1. There exists a quantifier $Q$ generating the weighting vector $\boldsymbol{e}_{1}$ such that $W_{\boldsymbol{p}, \boldsymbol{e}_{1}}^{Q}=\max$ if and only if $\min (\boldsymbol{p})>0$. 
2. There exists a quantifier $Q$ generating the weighting vector $\boldsymbol{e}_{n}$ such that $W_{\boldsymbol{p}, \boldsymbol{e}_{n}}^{Q}=\min$ if and only if $\min (\boldsymbol{p})>0$.

We have seen that, when $\boldsymbol{w}=\boldsymbol{e}_{l}$, we can recover the order statistic $O_{\boldsymbol{e}_{l}}$ from the WOWA operator if the weighting vector $\boldsymbol{p}$ satisfies a certain condition. However, as we can see in the following proposition, it is also possible to obtain a projection when we consider other weighting vectors $\boldsymbol{p}$.

Proposition 4. Let $l \in N$ and let $\boldsymbol{p}$ be a weighting vector. If there exists $k \in N$ such that $p_{k} \geq \max (l / n, 1-(l-1) / n)$, then $W_{\boldsymbol{p}, \boldsymbol{e}_{l}}^{Q}=M_{\boldsymbol{e}_{k}}$ for any quantifier $Q$ generating the weighting vector $\boldsymbol{e}_{l}$.

Proof. We are going to show that $\mu_{\boldsymbol{p}, \boldsymbol{e}_{l}}^{Q}=\mu_{\boldsymbol{e}_{k}}$. Notice that if $Q$ is a quantifier generating the weighting vector $\boldsymbol{e}_{l}$, then $Q(x)=0$ if $x \leq(l-1) / n$ and $Q(x)=1$ if $x \geq l / n$. Therefore, given $A \subseteq N$, we have

$$
\mu_{\boldsymbol{p}, e_{l}}^{Q}(A)= \begin{cases}0 & \text { if } \sum_{i \in A} p_{i} \leq(l-1) / n \\ Q\left(\sum_{i \in A} p_{i}\right) & \text { if }(l-1) / n<\sum_{i \in A} p_{i}<l / n \\ 1 & \text { if } \sum_{i \in A} p_{i} \geq l / n\end{cases}
$$

Suppose now that there exists $k \in N$ such that $p_{k} \geq \max (l / n, 1-(l-1) / n)$. We distinguish two cases:

1. If $k \notin A$, since $p_{k} \geq 1-(l-1) / n$, then $\sum_{i \in A} p_{i}=1-\sum_{i \notin A} p_{i} \leq 1-p_{k} \leq$ $(l-1) / n$ and, consequently, $\mu_{\boldsymbol{p}, \boldsymbol{e}_{l}}^{Q}(A)=0$.

2. If $k \in A$, since $p_{k} \geq l / n$, then $\sum_{i \in A} p_{i} \geq l / n$ and, consequently, $\mu_{\boldsymbol{p}, e_{l}}^{Q}(A)=$ 1 .

So, according to the first item of Remark $6, \mu_{\boldsymbol{e}_{k}, \boldsymbol{w}}^{Q}=\mu_{\boldsymbol{e}_{k}}$.

Propositions $2-4$ reflect that, in a certain sense, WOWA operators grant more importance to the weighting vector $\boldsymbol{p}$ than to the weighting vector $\boldsymbol{w}$ : When $\boldsymbol{p}=$ $\boldsymbol{e}_{k}$, we always obtain the projection $M_{\boldsymbol{e}_{k}}$ whereas when $\boldsymbol{w}=\boldsymbol{e}_{l}$ we cannot always get the order statistic $O_{\boldsymbol{e}_{l}}$ and, in some cases, we obtain a projection $M_{\boldsymbol{e}_{k}}$. For instance, consider $\boldsymbol{p}=(0.6,0.4,0,0,0), \boldsymbol{w}=(0,0,1,0,0)$ and $\boldsymbol{x}=(10,5,6,4,4)$. According to Proposition 4 , for any quantifier $Q$ generating the weighting vector $\boldsymbol{e}_{3}$ we get $W_{\boldsymbol{p}, \boldsymbol{e}_{3}}^{Q}(\boldsymbol{x})=M_{\boldsymbol{e}_{1}}(\boldsymbol{x})=10$ despite the fact that the value 10 is associated with the weights $p_{1}=0.6$ and $w_{1}=0$ whereas the value 5 is associated with the weights $p_{2}=0.4$ and $w_{3}=1$ (the topic of the weights will be discussed further in the next section).

\subsection{SUOWA operators}

In the case of SUOWA operators, the following propositions show that when we consider the weighting vectors $\boldsymbol{p}=\boldsymbol{e}_{k}$ and $\boldsymbol{w}=\boldsymbol{e}_{l}$, the functions $M_{\boldsymbol{e}_{k}}$ and $O_{\boldsymbol{e}_{l}}$ can be recovered from SUOWA operators. Notice that this fact is possible independently of the other weighting vector considered. 
Proposition 5. If $k \in N$, then there exists $U \in \widetilde{\mathcal{U}}^{1 / n}$ such that $S_{\boldsymbol{e}_{k}, \boldsymbol{w}}=M_{\boldsymbol{e}_{k}}$ for any weighting vector $\boldsymbol{w}$.

Proof. Let $\boldsymbol{w}$ be a weighting vector. Notice that given $U \in \widetilde{\mathcal{U}}^{1 / n}$, the game associated with $\boldsymbol{e}_{k}, \boldsymbol{w}$, and $U$ is defined by

$$
v_{\boldsymbol{e}_{k}, \boldsymbol{w}}^{U}(A)= \begin{cases}|A| U\left(0, \frac{\mu_{|\boldsymbol{w}|}(A)}{|A|}\right) & \text { if } k \notin A, \\ |A| U\left(\frac{1}{|A|}, \frac{\mu_{|\boldsymbol{w}|}(A)}{|A|}\right) & \text { if } k \in A .\end{cases}
$$

Consider now the semiuninorm $U_{\text {minmax }}$ defined by

$$
U_{\text {minmax }}(x, y)= \begin{cases}\min (x, y) & \text { if } x<1 / n \text { or }(x, y) \in\{1 / n\} \times[0,1 / n] \\ \max (x, y) & \text { otherwise. }\end{cases}
$$

It is easy to check that $U_{\operatorname{minmax}} \in \widetilde{\mathcal{U}}^{1 / n}$ (in fact $U_{\text {minmax }} \in \mathcal{U}_{\mathrm{i}}^{1 / n}$ ) and that

$$
v_{\boldsymbol{e}_{k}, \boldsymbol{w}}^{U_{\operatorname{minax}}}(A)= \begin{cases}0 & \text { if } k \notin A \\ 1 & \text { if } k \in A\end{cases}
$$

Therefore, $v_{\boldsymbol{e}_{k}, \boldsymbol{w}}^{U_{\operatorname{minmax}}}$ is a capacity on $N$ and, taking into account the first item of Remark 6, $v_{\boldsymbol{e}_{k}, \boldsymbol{w}}^{U, \boldsymbol{m i n m a x}}=\mu_{\boldsymbol{e}_{k}}$.

Proposition 6. If $l \in N$, then there exists $U \in \widetilde{\mathcal{U}}^{1 / n}$ such that $S_{\boldsymbol{p}, \boldsymbol{e}_{l}}^{U}=O_{\boldsymbol{e}_{l}}$ for any weighting vector $\boldsymbol{p}$.

Proof. Let $\boldsymbol{p}$ be a weighting vector. Notice that given $U \in \widetilde{\mathcal{U}}^{1 / n}$, the game associated with $\boldsymbol{p}, \boldsymbol{e}_{l}$, and $U$ is defined by

$$
v_{\boldsymbol{p}, \boldsymbol{e}_{l}}^{U}(A)= \begin{cases}|A| U\left(\frac{\mu_{\boldsymbol{p}}(A)}{|A|}, 0\right) & \text { if }|A|<l, \\ |A| U\left(\frac{\mu_{\boldsymbol{p}}(A)}{|A|}, \frac{1}{|A|}\right) & \text { if }|A| \geq l .\end{cases}
$$

Consider now the semiuninorm $U_{\min }^{\max }$ defined by

$$
U_{\min }^{\max }(x, y)= \begin{cases}\min (x, y) & \text { if } y<1 / n \text { or }(x, y) \in[0,1 / n] \times\{1 / n\}, \\ \max (x, y) & \text { otherwise }\end{cases}
$$

It is easy to check that $U_{\min }^{\max } \in \widetilde{\mathcal{U}}^{1 / n}$ (in fact $U_{\min }^{\max } \in \mathcal{U}_{\mathrm{i}}^{1 / n}$ ) and that

$$
v_{\boldsymbol{p}, e_{l}}^{U_{\min }^{\max }}(A)= \begin{cases}0 & \text { if }|A|<l \\ 1 & \text { if }|A| \geq l\end{cases}
$$

Therefore, $v_{\substack{\min \\ U_{\operatorname{en}}}}^{U_{\max }^{\max }}$ is a capacity on $N$ and, taking into account the second item of Remark 6, $v_{\boldsymbol{p}, \boldsymbol{e}_{l}}^{U_{\operatorname{mix}}^{\max }}=\mu_{\left|\boldsymbol{e}_{l}\right|}$. 
In the sequel, we put both conditions together and study the case $\boldsymbol{p}=\boldsymbol{e}_{k}$ and $\boldsymbol{w}=\boldsymbol{e}_{l}$. Notice that when $k, l \in N$ and $U \in \widetilde{\mathcal{U}}^{1 / n}$, the game associated with $\boldsymbol{e}_{k}, \boldsymbol{e}_{l}$, and $U$ is defined by

$$
v_{\boldsymbol{e}_{k}, \boldsymbol{e}_{l}}^{U}(A)= \begin{cases}0 & \text { if } k \notin A \text { and }|A|<l, \\ |A| U\left(0, \frac{1}{|A|}\right) & \text { if } k \notin A \text { and }|A| \geq l, \\ |A| U\left(\frac{1}{|A|}, 0\right) & \text { if } k \in A \text { and }|A|<l, \\ 1 & \text { if } k \in A \text { and }|A| \geq l,\end{cases}
$$

where the last case, $v_{\boldsymbol{e}_{k}, \boldsymbol{e}_{l}}^{U}(A)=1$ when $k \in A$ and $|A| \geq l$, is obtained from the fact that

$$
1=|A| \max \left(\frac{1}{|A|}, \frac{1}{|A|}\right) \leq|A| U\left(\frac{1}{|A|}, \frac{1}{|A|}\right) \leq|A| \frac{1}{|A|}=1 .
$$

In the specific cases of $U_{\perp}, U_{\top}, U_{\min }$, and $U_{\max }$ we get

$$
\begin{aligned}
& v_{\boldsymbol{e}_{k}, \boldsymbol{e}_{l}}^{U_{\perp}}(A)=v_{\boldsymbol{e}_{k}, \boldsymbol{e}_{l}}^{U_{\min }}(A)= \begin{cases}0 & \text { if } k \notin A \text { or }|A|<l, \\
1 & \text { if } k \in A \text { and }|A| \geq l,\end{cases} \\
& v_{\boldsymbol{e}_{k}, \boldsymbol{e}_{l}}^{U_{\top}}(A)=v_{\boldsymbol{e}_{k}, \boldsymbol{e}_{l}}^{U_{\max }}(A)= \begin{cases}0 & \text { if } k \notin A \text { and }|A|<l, \\
1 & \text { if } k \in A \text { or }|A| \geq l,\end{cases}
\end{aligned}
$$

and it is easy to check that both games are capacities. In the following proposition, we show which are the SUOWA operators associated with these capacities.

Proposition 7. Let $k, l \in N$. Then, given $\boldsymbol{x} \in \mathbb{R}^{n}, S_{\boldsymbol{e}_{k}, \boldsymbol{e}_{l}}^{U_{\perp}}(\boldsymbol{x})=S_{\boldsymbol{e}_{k}, \boldsymbol{e}_{l}}^{U_{\min }}(\boldsymbol{x})=$ $\min \left(x_{k}, x_{[l]}\right)$ and $S_{\boldsymbol{e}_{k}, \boldsymbol{e}_{l}}^{U_{\top}}(\boldsymbol{x})=S_{\boldsymbol{e}_{k}, \boldsymbol{e}_{l}}^{U_{\max }}(\boldsymbol{x})=\max \left(x_{k}, x_{[l]}\right)$.

Proof. Let $\boldsymbol{x} \in \mathbb{R}^{n}$ and consider $U_{\text {min }}$. First, we are going to see that

$$
S_{\boldsymbol{e}_{k}, \boldsymbol{e}_{l}}^{U_{\min }}(\boldsymbol{x})= \begin{cases}x_{[l]} & \text { if } k \in A_{[l]}, \\ x_{k} & \text { if } k \notin A_{[l]} .\end{cases}
$$

We distinguish two cases:

1. If $k \in A_{[l]}$, then $v_{\boldsymbol{e}_{k}, e_{l}}^{U_{\min }}\left(A_{[l]}\right)=1$ and $v_{\boldsymbol{e}_{k}, \boldsymbol{e}_{l}}^{U_{\min }}\left(A_{[l-1]}\right)=0$. Therefore, according to expression $(2), S_{\boldsymbol{e}_{k}, \boldsymbol{e}_{l}}^{U_{\min }}(\boldsymbol{x})=x_{[l]}$.

2. If $k \notin A_{[l]}$, let $j \in N$ such that $[j]=k$. Then $v_{\boldsymbol{e}_{k}, \boldsymbol{e}_{l}}^{U_{\min }}\left(A_{[j]}\right)=1$ and $v_{\boldsymbol{e}_{k}, \boldsymbol{e}_{l}}^{U_{\min }}\left(A_{[j-1]}\right)=0$. Therefore, according to expression $(2), S_{\boldsymbol{e}_{k}, \boldsymbol{e}_{l}}^{U_{\min }}(\boldsymbol{x})=x_{[j]}=$ $x_{k}$.

Now, let us see that $S_{\boldsymbol{e}_{k}, \boldsymbol{e}_{l}}^{U_{\min }}(\boldsymbol{x})=\min \left(x_{k}, x_{[l]}\right)$. We distinguish three cases:

1. If $x_{k}>x_{[l]}$, then $k \in A_{[l]}$ and, consequently, $S_{\boldsymbol{e}_{k}, \boldsymbol{e}_{l}}^{U_{\min }}(\boldsymbol{x})=x_{[l]}=\min \left(x_{k}, x_{[l]}\right)$. 
2. If $x_{k}=x_{[l]}$, then, obviously, $S_{\boldsymbol{e}_{k}, \boldsymbol{e}_{l}}^{U_{\min }}(\boldsymbol{x})=\min \left(x_{k}, x_{[l]}\right)$.

3. If $x_{k}<x_{[l]}$, then $k \notin A_{[l]}$ and, consequently, $S_{\boldsymbol{e}_{k}, \boldsymbol{e}_{l}}^{U_{\min }}(\boldsymbol{x})=x_{k}=\min \left(x_{k}, x_{[l]}\right)$.

The statement $S_{\boldsymbol{e}_{k}, \boldsymbol{e}_{l}}^{U_{\top}}(\boldsymbol{x})=S_{\boldsymbol{e}_{k}, \boldsymbol{e}_{l}}^{U_{\max }}(\boldsymbol{x})=\max \left(x_{k}, x_{[l]}\right)$ can be proven in a similar way.

As an immediate consequence of Proposition 1, we get the following results. Corollary 2. Let $k, l \in N$ and $U \in \widetilde{\mathcal{U}}^{1 / n}$. Then the following holds:

1. Given $\boldsymbol{x} \in \mathbb{R}^{n}, \min \left(x_{k}, x_{[l]}\right) \leq S_{\boldsymbol{e}_{k}, \boldsymbol{e}_{l}}^{U}(\boldsymbol{x}) \leq \max \left(x_{k}, x_{[l]}\right)$.

2. If $U_{\perp} \leq U \leq U_{\min }$, then $S_{\boldsymbol{e}_{k}, \boldsymbol{e}_{l}}^{U}(\boldsymbol{x})=\min \left(x_{k}, x_{[l]}\right)$ for all $\boldsymbol{x} \in \mathbb{R}^{n}$.

3. If $U_{\max } \leq U \leq U_{\mathrm{T}}$, then $S_{\boldsymbol{e}_{k}, \boldsymbol{e}_{l}}^{U}(\boldsymbol{x})=\max \left(x_{k}, x_{[l]}\right)$ for all $\boldsymbol{x} \in \mathbb{R}^{n}$.

Some specific cases are given in the following corollary.

Corollary 3. Let $U \in \widetilde{\mathcal{U}}^{1 / n}$. Then the following holds:

1. $M_{\boldsymbol{e}_{1}} \leq S_{\boldsymbol{e}_{1}, \boldsymbol{e}_{1}}^{U} \leq O_{\boldsymbol{e}_{1}}, S_{\boldsymbol{e}_{1}, \boldsymbol{e}_{1}}^{U}=M_{\boldsymbol{e}_{1}}$ if $U_{\perp} \leq U \leq U_{\min }$, and $S_{\boldsymbol{e}_{1}, \boldsymbol{e}_{1}}^{U}=O_{\boldsymbol{e}_{1}}$ if $U_{\max } \leq U \leq U_{\mathrm{T}}$.

2. $O_{\boldsymbol{e}_{n}} \leq S_{\boldsymbol{e}_{n}, \boldsymbol{e}_{n}}^{U} \leq M_{\boldsymbol{e}_{n}}$, $S_{\boldsymbol{e}_{n}, \boldsymbol{e}_{n}}^{U}=O_{\boldsymbol{e}_{n}}$ if $U_{\perp} \leq U \leq U_{\min }$, and $S_{\boldsymbol{e}_{n}, \boldsymbol{e}_{n}}^{U}=M_{\boldsymbol{e}_{n}}$ if $U_{\max } \leq U \leq U_{\mathrm{T}}$

\section{CAPACITIES, WEIGHTS, AND VALUES}

WOWA and SUOWA operators are Choquet integrals that generalize weighted means and OWA operators. Hence, they are depicted through the capacities from which they are building or the weights affecting the components of $\boldsymbol{x}$ (see expressions (1) and (2)). Moreover, from a behavioral perspective, it is also important to analyze the values they return. So, it seems very interesting to know the relationship between these features of WOWA and SUOWA operators and the corresponding ones of weighted means and OWA operators. The aim of this section is to analyze WOWA and SUOWA operators from these three points of view: the capacities, the weights, and the values they return.

\subsection{The capacities}

As we have seen, the notion of capacity is essential in the definition of the Choquet integral. Given $A \subseteq N$, the value $\mu(A)$ can be seen as the "weight" related to the subset of criteria $A .{ }^{31}$ Given that WOWA and SUOWA operators have appeared in the literature as an answer to the problem of combining weighted means and OWA operators in a single function, it seems suitable to analyze the capacities of WOWA and SUOWA operators in relation to those of weighted means and OWA operators. In the case of SUOWA operators, we have the following properties. 
Proposition 8 (Llamazares $\left.{ }^{8}\right)$. Let $\boldsymbol{p}$ and $\boldsymbol{w}$ be two weighting vectors, and $U \in$ $\mathcal{U}_{\mathrm{i}}^{1 / n}$. Given $A \subseteq N$, the following holds:

1. $\min \left(\mu_{\boldsymbol{p}}(A), \mu_{|\boldsymbol{w}|}(A)\right) \leq \hat{v}_{\boldsymbol{p}, \boldsymbol{w}}^{U}(A) \leq \max \left(\mu_{\boldsymbol{p}}(A), \mu_{|\boldsymbol{w}|}(A)\right)$.

2. If $\mu_{\boldsymbol{p}}(A)=\mu_{|\boldsymbol{w}|}(A)$, then $\hat{v}_{\boldsymbol{p}, \boldsymbol{w}}^{U}(A)=\mu_{\boldsymbol{p}}(A)$.

Hence, when we consider idempotent semiuninorms, the capacity associated with a SUOWA operator ranges between the capacities of the corresponding weighted mean and OWA operator. Moreover, when both capacities take the same value on a set, the capacity associated with the SUOWA operator coincides with them. As we can see in the following example, WOWA operators do not satisfy these properties.

Example 1. Consider the weighting vectors $\boldsymbol{p}=(0.3,0.3,0.2,0.1,0.1)$ and $\boldsymbol{w}=$ $(0.4,0.3,0.1,0.1,0.1)$ and $A=\{1,2,3\}$. It is easy to check that $\mu_{\boldsymbol{p}}(A)=$ $\mu_{|\boldsymbol{w}|}(A)=0.8$. Now, if $Q$ is a quantifier generating the weighting vector $\boldsymbol{w}$, then $Q(0)=0, Q(0.2)=0.4, Q(0.4)=0.7, Q(0.6)=0.8, Q(0.8)=0.9$, and $Q(1)=1$. Consequently, $\mu_{\boldsymbol{p}, \boldsymbol{w}}^{Q}(A)=Q\left(\mu_{\boldsymbol{p}}(A)\right)=Q(0.8)=0.9$.

\subsection{The weights}

Weighted means and OWA operators are functions defined through weighting vectors. So, given $\boldsymbol{x} \in \mathbb{R}^{n}$, the element $x_{[i]}$ is weighted by the value $p_{[i]}$ in the case of weighted means while it is weighted by the value $w_{i}$ in the case of OWA operators. Hence, a natural question is the relationship between the weights associated with $x_{[i]}$ in the case of WOWA and SUOWA operators, $q_{i}$ and $s_{i}$ respectively, and the weights $p_{[i]}$ and $w_{i}$. As we show in the following results, ${ }^{\mathrm{c}}$ in the case of SUOWA operators and under certain hypothesis on $\boldsymbol{p}, \boldsymbol{w}$, and $U$, we can provide some interesting information on the weight $s_{i}$. For instance, in some cases, the weight $s_{i}$ can be straightforward obtained from $p_{[i]}$ and $w_{i}$.

Proposition 9. Let $\boldsymbol{p}$ and $\boldsymbol{w}$ be two weighting vectors such that $\sum_{i=1}^{j} w_{i} \leq j / n$ for all $j \in N$ and $\min _{i \in N} p_{i}+\min _{i \in N} w_{i} \geq 1 / n$. Then the following holds:

1. For any $A \subseteq N$ such that $1 \leq|A| \leq n$ we have

$$
v_{\boldsymbol{p}, \boldsymbol{w}}^{U_{T_{\mathbf{L}}}}(A)=\mu_{\boldsymbol{p}}(A)+\mu_{|\boldsymbol{w}|}(A)-\frac{|A|}{n}=\sum_{i \in A} p_{i}+\sum_{i=1}^{|A|} w_{i}-\frac{|A|}{n} .
$$

2. $v_{\boldsymbol{p}, \boldsymbol{w}}^{U_{T_{\mathbf{L}}}}$ is a capacity on $N$.

3. Given $\boldsymbol{x} \in \mathbb{R}^{n}, s_{i}^{U_{T_{\mathbf{L}}}}=p_{[i]}+w_{i}-1 / n$ for any $i \in N$, and, consequently, $S_{\boldsymbol{p}, \boldsymbol{w}}^{U_{T_{\mathbf{L}}}}(\boldsymbol{x})=M_{\boldsymbol{p}}(\boldsymbol{x})+O_{\boldsymbol{w}}(\boldsymbol{x})-\overline{\boldsymbol{x}}$ (where $\overline{\boldsymbol{x}}$ is the average of $\boldsymbol{x}$ ).

${ }^{\mathrm{c}}$ In some results, we will use the notation $s_{i}^{U}$ to indicate that we are employing the semiuninorm $U$ for obtaining the weight $s_{i}$. 
Proof.

1. Let $A \subseteq N$ be such that $1 \leq|A| \leq n$. Since $\left(\sum_{i=1}^{j} w_{i}\right) / j \leq 1 / n$ for all $j \in N$, and

$$
\frac{\mu_{\boldsymbol{p}}(A)}{|A|}+\frac{\mu_{|\boldsymbol{w}|}(A)}{|A|}=\frac{\sum_{i \in A} p_{i}}{|A|}+\frac{\sum_{i=1}^{|A|} w_{i}}{|A|} \geq \min _{i \in N} p_{i}+\min _{i \in N} w_{i} \geq \frac{1}{n},
$$

we have

$$
\begin{aligned}
v_{\boldsymbol{p}, \boldsymbol{w}}^{U_{T_{\mathbf{L}}}}(A) & =|A| U_{T_{\mathbf{L}}}\left(\frac{\mu_{\boldsymbol{p}}(A)}{|A|}, \frac{\mu_{|\boldsymbol{w}|}(A)}{|A|}\right)=\mu_{\boldsymbol{p}}(A)+\mu_{|\boldsymbol{w}|}(A)-\frac{|A|}{n} \\
& =\sum_{i \in A} p_{i}+\sum_{i=1}^{|A|} w_{i}-\frac{|A|}{n} .
\end{aligned}
$$

2. Consider $B \subsetneq A \subseteq N$. Since $\min _{i \in N} p_{i}+\min _{i \in N} w_{i}-1 / n \geq 0$, we have

$$
\sum_{i \in A \backslash B} p_{i}+\sum_{i=|B|+1}^{|A|} w_{i}-\frac{|A|-|B|}{n} \geq 0 .
$$

Therefore,

$$
\begin{aligned}
v_{\boldsymbol{p}, \boldsymbol{w}}^{U_{T_{\mathbf{L}}}}(B)= & \sum_{i \in B} p_{i}+\sum_{i=1}^{|B|} w_{i}-\frac{|B|}{n} \leq \sum_{i \in B} p_{i}+\sum_{i=1}^{|B|} w_{i}-\frac{|B|}{n}+\sum_{i \in A \backslash B} p_{i}+\sum_{i=|B|+1}^{|A|} w_{i} \\
& -\frac{|A|-|B|}{n}=\sum_{i \in A} p_{i}+\sum_{i=1}^{|A|} w_{i}-\frac{|A|}{n}=v_{\boldsymbol{p}, \boldsymbol{w}}^{U_{T_{\mathbf{L}}}}(A)
\end{aligned}
$$

that is, $v_{\boldsymbol{p}, \boldsymbol{w}}^{U_{\boldsymbol{w}_{\mathbf{L}}}}$ is a capacity on $N$.

3. Given $\boldsymbol{x} \in \mathbb{R}^{n}$, for any $i \in N$ we have

$$
\begin{aligned}
s_{i}^{U_{T_{\mathbf{L}}}} & =v_{\boldsymbol{p}, \boldsymbol{w}}^{U_{T_{\mathbf{L}}}}\left(A_{[i]}\right)-v_{\boldsymbol{p}, \boldsymbol{w}}^{U_{T_{\mathbf{L}}}}\left(A_{[i-1]}\right) \\
& =\sum_{j=1}^{i} p_{[j]}+\sum_{j=1}^{i} w_{j}-\frac{i}{n}-\sum_{j=1}^{i-1} p_{[j]}-\sum_{j=1}^{i-1} w_{j}+\frac{i-1}{n}=p_{[i]}+w_{i}-1 / n .
\end{aligned}
$$

Hence,

$$
S_{\boldsymbol{p}, \boldsymbol{w}}^{U_{T_{\mathbf{L}}}}(\boldsymbol{x})=\sum_{i=1}^{n}\left(p_{[i]}+w_{i}-1 / n\right) x_{[i]}=M_{\boldsymbol{p}}(\boldsymbol{x})+O_{\boldsymbol{w}}(\boldsymbol{x})-\overline{\boldsymbol{x}} .
$$

Idempotent semiuninorms allow us to obtain the following result. 
Proposition 10 (Llamazares $^{8}$ ). Let $\boldsymbol{p}$ and $\boldsymbol{w}$ be two weighting vectors, let $U \in$ $\mathcal{U}_{\mathrm{i}}^{1 / n}$, and let $\boldsymbol{x} \in \mathbb{R}^{n}$ such that $p_{[i]}=w_{i}$ for all $i \in N$. Then $s_{i}=p_{[i]}=w_{i}$ for all $i \in N$ (and, consequently, $\left.S_{\boldsymbol{p}, \boldsymbol{w}}^{U}(\boldsymbol{x})=M_{\boldsymbol{p}}(\boldsymbol{x})=O_{\boldsymbol{w}}(\boldsymbol{x})\right)$.

Therefore, given $\boldsymbol{x} \in \mathbb{R}^{n}$, if the weights $p_{[i]}$ and $w_{i}$ are equal for all $i \in N$ and we consider idempotent semiuninorms, then the corresponding weights $s_{i}$ of the SUOWA operator coincide with them. Notice that this property is not satisfied in the case of WOWA operators, as it has been made clear by Llamazares. ${ }^{12}$

Another interesting question about the weights $s_{i}$ is to know whether they are located between $p_{[i]}$ and $w_{i}$; that is, when $\min \left(p_{[i]}, w_{i}\right) \leq s_{i} \leq \max \left(p_{[i]}, w_{i}\right)$ for all $i \in N$. This condition can be guaranteed by considering some specific weighting vectors $\boldsymbol{w}$ and the uninorms $U_{\min }$ and $U_{\max }$. Before establishing these results, in Propositions 11 and 12, we recall some useful properties.

Proposition 11 (Llamazares $\left.{ }^{12}\right)$. Let $\boldsymbol{w}$ be a weighting vector with $\sum_{i=1}^{j} w_{i}<$ $j / n$ for all $j \in\{1, \ldots, n-1\}$. Then, for all weighting vector $\boldsymbol{p}$, we have

1. $v_{\boldsymbol{p}, \boldsymbol{w}}^{U_{\min }}$ is a capacity on $N$.

2. $v_{\boldsymbol{p}, \boldsymbol{w}}^{U_{\min }}(A)=\min \left(\mu_{\boldsymbol{p}}(A), \mu_{|\boldsymbol{w}|}(A)\right)$ for all $A \subseteq N$.

3. $S_{\boldsymbol{p}, \boldsymbol{w}}^{U_{\min }}(\boldsymbol{x}) \leq \min \left(M_{\boldsymbol{p}}(\boldsymbol{x}), O_{\boldsymbol{w}}(\boldsymbol{x})\right)$ for all $\boldsymbol{x} \in \mathbb{R}^{n}$.

Proposition 12 (Llamazares $\left.{ }^{12}\right)$. Let $\boldsymbol{w}$ be a weighting vector with $\sum_{i=1}^{j} w_{i}>$ $j / n$ for all $j \in\{1, \ldots, n-1\}$. Then, for all weighting vector $\boldsymbol{p}$, we get

1. $v_{\boldsymbol{p}, \boldsymbol{w}}^{U_{\max }}$ is a capacity on $N$.

2. $v_{\boldsymbol{p}, \boldsymbol{w}}^{U_{\max }}(A)=\max \left(\mu_{\boldsymbol{p}}(A), \mu_{|\boldsymbol{w}|}(A)\right)$ for all $A \subseteq N$.

3. $S_{\boldsymbol{p}, \boldsymbol{w}}^{U_{\max }}(\boldsymbol{x}) \geq \max \left(M_{\boldsymbol{p}}(\boldsymbol{x}), O_{\boldsymbol{w}}(\boldsymbol{x})\right)$ for all $\boldsymbol{x} \in \mathbb{R}^{n}$.

Proposition 13. Let $\boldsymbol{w}$ be a weighting vector such that $\sum_{i=1}^{j} w_{i}<j / n$ for all $j \in\{1, \ldots, n-1\}$. Then, for any weighting vector $\boldsymbol{p}$, for any $\boldsymbol{x} \in \mathbb{R}^{n}$ and for any $i \in N$, we have $\min \left(p_{[i]}, w_{i}\right) \leq s_{i}^{U_{\min }} \leq \max \left(p_{[i]}, w_{i}\right)$.

Proof. Given $i \in N$, by Proposition 11 we get $s_{i}^{U_{\min }}=\min \left(\mu_{\boldsymbol{p}}\left(A_{[i]}\right), \mu_{|\boldsymbol{w}|}\left(A_{[i]}\right)\right)-$ $\min \left(\mu_{\boldsymbol{p}}\left(A_{[i-1]}\right), \mu_{|\boldsymbol{w}|}\left(A_{[i-1]}\right)\right)$. We distinguish four cases:

1. If $\mu_{\boldsymbol{p}}\left(A_{[i]}\right) \leq \mu_{|\boldsymbol{w}|}\left(A_{[i]}\right)$ and $\mu_{\boldsymbol{p}}\left(A_{[i-1]}\right) \leq \mu_{|\boldsymbol{w}|}\left(A_{[i-1]}\right)$, then

$$
s_{i}^{U_{\min }}=\mu_{\boldsymbol{p}}\left(A_{[i]}\right)-\mu_{\boldsymbol{p}}\left(A_{[i-1]}\right)=p_{[i]} .
$$

2. If $\mu_{\boldsymbol{p}}\left(A_{[i]}\right) \leq \mu_{|\boldsymbol{w}|}\left(A_{[i]}\right)$ and $\mu_{\boldsymbol{p}}\left(A_{[i-1]}\right)>\mu_{|\boldsymbol{w}|}\left(A_{[i-1]}\right)$, then $s_{i}^{U_{\min }}=\mu_{\boldsymbol{p}}\left(A_{[i]}\right)-$ $\mu_{|\boldsymbol{w}|}\left(A_{[i-1]}\right)$, and notice that

$$
w_{i}=\mu_{|\boldsymbol{w}|}\left(A_{[i]}\right)-\mu_{|\boldsymbol{w}|}\left(A_{[i-1]}\right) \geq s_{i}^{U_{\min }}>\mu_{\boldsymbol{p}}\left(A_{[i]}\right)-\mu_{\boldsymbol{p}}\left(A_{[i-1]}\right)=p_{[i]} .
$$


3. If $\mu_{\boldsymbol{p}}\left(A_{[i]}\right)>\mu_{|\boldsymbol{w}|}\left(A_{[i]}\right)$ and $\mu_{\boldsymbol{p}}\left(A_{[i-1]}\right) \leq \mu_{|\boldsymbol{w}|}\left(A_{[i-1]}\right)$, then we have $s_{i}^{U_{\min }}=$ $\mu_{|\boldsymbol{w}|}\left(A_{[i]}\right)-\mu_{\boldsymbol{p}}\left(A_{[i-1]}\right)$, and note that

$$
p_{[i]}=\mu_{\boldsymbol{p}}\left(A_{[i]}\right)-\mu_{\boldsymbol{p}}\left(A_{[i-1]}\right)>s_{i}^{U_{\min }} \geq \mu_{|\boldsymbol{w}|}\left(A_{[i]}\right)-\mu_{|\boldsymbol{w}|}\left(A_{[i-1]}\right)=w_{i} .
$$

4. If $\mu_{\boldsymbol{p}}\left(A_{[i]}\right)>\mu_{|\boldsymbol{w}|}\left(A_{[i]}\right)$ and $\mu_{\boldsymbol{p}}\left(A_{[i-1]}\right)>\mu_{|\boldsymbol{w}|}\left(A_{[i-1]}\right)$, then

$$
s_{i}^{U_{\min }}=\mu_{|\boldsymbol{w}|}\left(A_{[i]}\right)-\mu_{|\boldsymbol{w}|}\left(A_{[i-1]}\right)=w_{i} .
$$

So, in any of the four cases, the condition $\min \left(p_{[i]}, w_{i}\right) \leq s_{i}^{U_{\min }} \leq \max \left(p_{[i]}, w_{i}\right)$ is satisfied.

A similar result can be established in the case of the uninorm $U_{\max }$ (the proof is omitted because it is similar to that of Proposition 13).

Proposition 14. Let $\boldsymbol{w}$ be a weighting vector such that $\sum_{i=1}^{j} w_{i}>j / n$ for all $j \in\{1, \ldots, n-1\}$. Then, for any weighting vector $\boldsymbol{p}$, for any $\boldsymbol{x} \in \mathbb{R}^{n}$ and for any $i \in N$, we have $\min \left(p_{[i]}, w_{i}\right) \leq s_{i}^{U_{\max }} \leq \max \left(p_{[i]}, w_{i}\right)$.

Another interesting property is obtained when we consider convex combinations of semiuninorms and the games associated with the semiuninorms are normalized capacities. Then, given $\boldsymbol{x} \in \mathbb{R}^{n}$, the weight associated with $x_{[i]}$ (for the SUOWA operator associated with the new semiuninorm) can be straightforward obtained by using the same convex combination of the weights associated with $x_{[i]}$ in the SUOWA operators associated with the former semiuninorms. Before establishing the result, in the following proposition we recall some useful properties of convex combinations of semiuninorms.

Proposition 15 (Llamazares ${ }^{12}$ ). Let $\boldsymbol{p}$ and $\boldsymbol{w}$ be two weighting vectors, let $U_{1}, \ldots, U_{m} \in \widetilde{\mathcal{U}}^{1 / n}$ such that $v_{\boldsymbol{p}, \boldsymbol{w}}^{U_{1}}, \ldots, v_{\boldsymbol{p}, \boldsymbol{w}}^{U_{m}}$ be normalized capacities, let $\boldsymbol{\lambda} \in$ $[0,1]^{m}$ be a weighting vector, and let $U=\sum_{j=1}^{m} \lambda_{j} U_{j}$. Then the following holds:

1. $U \in \widetilde{\mathcal{U}}^{1 / n}$, and if $U_{1}, \ldots, U_{m} \in \mathcal{U}_{\mathrm{i}}^{1 / n}$, then $U \in \mathcal{U}_{\mathrm{i}}^{1 / n}$.

2. $v_{\boldsymbol{p}, \boldsymbol{w}}^{U}(A)=\sum_{j=1}^{m} \lambda_{j} v_{\boldsymbol{p}, \boldsymbol{w}}^{U_{j}}(A)$ for any subset $A$ of $N$.

3. $v_{\boldsymbol{p}, \boldsymbol{w}}^{U}$ is a normalized capacity.

4. $S_{\boldsymbol{p}, \boldsymbol{w}}^{U}=\sum_{j=1}^{m} \lambda_{j} S_{\boldsymbol{p}, \boldsymbol{w}}^{U_{j}}$.

Proposition 16. Let $\boldsymbol{p}$ and $\boldsymbol{w}$ be two weighting vectors, let $U_{1}, \ldots, U_{m} \in \widetilde{\mathcal{U}}^{1 / n}$ such that $v_{\boldsymbol{p}, \boldsymbol{w}}^{U_{1}}, \ldots, v_{\boldsymbol{p}, \boldsymbol{w}}^{U_{m}}$ be normalized capacities, let $\boldsymbol{\lambda} \in[0,1]^{m}$ be a weighting vector, and let $U=\sum_{j=1}^{m} \lambda_{j} U_{j}$. Then, for any $\boldsymbol{x} \in \mathbb{R}^{n}$ and for any $i \in N$, we have $s_{i}^{U}=\sum_{j=1}^{m} \lambda_{j} s_{i}^{U_{j}}$. 
Proof. Let $\boldsymbol{x} \in \mathbb{R}^{n}$ and $i \in N$. By Proposition 15, we get

$$
\begin{aligned}
s_{i}^{U} & =v_{\boldsymbol{p}, \boldsymbol{w}}^{U}\left(A_{[i]}\right)-v_{\boldsymbol{p}, \boldsymbol{w}}^{U}\left(A_{[i-1]}\right)=\sum_{j=1}^{m} \lambda_{j} v_{\boldsymbol{p}, \boldsymbol{w}}^{U_{j}}\left(A_{[i]}\right)-\sum_{j=1}^{m} \lambda_{j} v_{\boldsymbol{p}, \boldsymbol{w}}^{U_{j}}\left(A_{[i-1]}\right) \\
& =\sum_{j=1}^{m} \lambda_{j}\left(v_{\boldsymbol{p}, \boldsymbol{w}}^{U_{j}}\left(A_{[i]}\right)-v_{\boldsymbol{p}, \boldsymbol{w}}^{U_{j}}\left(A_{[i-1]}\right)\right)=\sum_{j=1}^{m} \lambda_{j} s_{i}^{U_{j}} .
\end{aligned}
$$

Obviously, if all the weights $s_{i}^{U_{j}}$ are located between $p_{[i]}$ and $w_{i}$, then so does the convex combination of them.

COROllary 4. Let $\boldsymbol{p}$ and $\boldsymbol{w}$ be two weighting vectors, let $U_{1}, \ldots, U_{m} \in \widetilde{\mathcal{U}}^{1 / n}$ such that $v_{\boldsymbol{p}, \boldsymbol{w}}^{U_{1}}, \ldots, v_{\boldsymbol{p}, \boldsymbol{w}}^{U_{m}}$ be normalized capacities, let $\boldsymbol{\lambda} \in[0,1]^{m}$ be a weighting vector, and let $U=\sum_{j=1}^{m} \lambda_{j} U_{j}$. Given $\boldsymbol{x} \in \mathbb{R}^{n}$ and $i \in N$, if $\min \left(p_{[i]}, w_{i}\right) \leq$ $s_{i}^{U_{j}} \leq \max \left(p_{[i]}, w_{i}\right)$ for all $j \in\{1, \ldots, m\}$ then we also get $\min \left(p_{[i]}, w_{i}\right) \leq s_{i}^{U} \leq$ $\max \left(p_{[i]}, w_{i}\right)$.

In the sequel, and using some of the previous results, we illustrate how we can get a SUOWA operator having a specific weight $s_{i}$.

Example 2. Let us consider the weighting vectors $\boldsymbol{p}=(0.1,0.4,0.4,0.1)$ and $\boldsymbol{w}=(0.2,0.2,0.3,0.3)$, and the idempotent uninorms $U_{\min }$ and $U_{\max }$. In the case of $U_{\min }$, the first item of Proposition 11 guarantees that $v_{\boldsymbol{p}, \boldsymbol{w}}^{U_{\min }}$ is a capacity. As we can see in Table 1 , this is also the case when we consider the uninorm $U_{\max }$.

Consider now $\boldsymbol{x}=(5,8,10,7)$. In the case of $U_{\text {min }}$, Proposition 13 guarantees that $\min \left(p_{[i]}, w_{i}\right) \leq s_{i}^{U_{\min }} \leq \max \left(p_{[i]}, w_{i}\right)$ for all $i \in N$. As we can see in Table 2, this is also the case when we consider the uninorm $U_{\max }$ (note that for this particular value of $\boldsymbol{x}$ we also have $s_{i}^{U_{\min }}=w_{i}$ and $s_{i}^{U_{\max }}=p_{[i]}$ for all $\left.i \in N\right)$.

So, according to Corollary 4 , any convex combination between $U_{\min }$ and $U_{\max }$ allows us to obtain weights located between $p_{[i]}$ and $w_{i}$. Moreover, notice that, by Proposition 16, we can easily obtain an idempotent semiuninorm that allows us to get a SUOWA operator having a specific weight $s_{i}^{U}$. For instance, if we look for an idempotent semi-uninorm $U$ such that $s_{1}^{U}=0.3$, it is sufficient to consider $U_{\mathrm{am}}=$ $0.5 U_{\min }+0.5 U_{\max }$, that is, the idempotent semi-uninorm obtained through the arithmetic mean:

$$
U_{\mathrm{am}}(x, y)= \begin{cases}\min (x, y) & \text { if }(x, y) \in[0,0.25]^{2} \\ \max (x, y) & \text { if }(x, y) \in[0.25,1]^{2} \backslash\{(0.25,0.25)\} \\ (x+y) / 2 & \text { otherwise }\end{cases}
$$

\subsection{The values}

A first question is whether the value returned by the WOWA operator and the SUOWA operator lies between the values returned by the weighted mean and the OWA operator. As we can see in the following example, this property is not generally satisfied. 
Table 1: Capacities associated with $U_{\min }$ and $U_{\max }$.

\begin{tabular}{lll}
\hline Set & $v_{\boldsymbol{p}, \boldsymbol{w}}^{U_{\min }}$ & $v_{\boldsymbol{p}, \boldsymbol{w}}^{U_{\max }}$ \\
\hline$\{1\}$ & 0.1 & 0.1 \\
$\{2\}$ & 0.2 & 0.4 \\
$\{3\}$ & 0.2 & 0.4 \\
$\{4\}$ & 0.1 & 0.1 \\
$\{1,2\}$ & 0.4 & 0.4 \\
$\{1,3\}$ & 0.4 & 0.4 \\
$\{1,4\}$ & 0.2 & 0.2 \\
$\{2,3\}$ & 0.4 & 0.8 \\
$\{2,4\}$ & 0.4 & 0.4 \\
$\{3,4\}$ & 0.4 & 0.4 \\
$\{1,2,3\}$ & 0.7 & 0.9 \\
$\{1,2,4\}$ & 0.6 & 0.6 \\
$\{1,3,4\}$ & 0.6 & 0.6 \\
$\{2,3,4\}$ & 0.7 & 0.9 \\
$N$ & 1 & 1 \\
\hline
\end{tabular}

Table 2: Weights associated with $x_{[i]}$, where $\boldsymbol{x}=(5,8,10,7)$.

\begin{tabular}{lllll}
\hline$i$ & $p_{[i]}$ & $w_{i}$ & $s_{i}^{U_{\min }}$ & $s_{i}^{U_{\max }}$ \\
\hline 1 & 0.4 & 0.2 & 0.2 & 0.4 \\
2 & 0.4 & 0.2 & 0.2 & 0.4 \\
3 & 0.1 & 0.3 & 0.3 & 0.1 \\
4 & 0.1 & 0.3 & 0.3 & 0.1 \\
\hline
\end{tabular}

Example 3. Consider $\boldsymbol{p}=(2 / 3,1 / 6,1 / 6), \boldsymbol{w}=(0.4,0.6,0)$ and $\boldsymbol{x}=(10,8,6)$. It is easy to check that $M_{\boldsymbol{p}}(\boldsymbol{x})=9$ and $O_{\boldsymbol{w}}(\boldsymbol{x})=8.8$. In the case of WOWA operators, and according to expression (3), given any quantifier $Q$ generating the weighting vector $\boldsymbol{w}$ we know that $Q(0)=0, Q(1 / 3)=0.4, Q(2 / 3)=1$ and $Q(1)=1$. Therefore, we have

$$
W_{\boldsymbol{p}, \boldsymbol{w}}^{Q}(\boldsymbol{x})=Q(2 / 3) \cdot 2+Q(5 / 6) \cdot 2+Q(1) \cdot 6=10,
$$

and, consequently, $W_{\boldsymbol{p}, \boldsymbol{w}}^{Q}(\boldsymbol{x})>\max \left(M_{\boldsymbol{p}}(\boldsymbol{x}), O_{\boldsymbol{w}}(\boldsymbol{x})\right)$. In the case of SUOWA operators, we calculate the capacity associated with the semi-uninorm $U_{\perp}$. Notice 
that, in this case, the capacity coincides with the game (see Table 3).

Table 3: Capacities associated with $U_{\perp}$ and $U_{\top}$.

\begin{tabular}{lll}
\hline Set & $\hat{v}_{\boldsymbol{p}, \boldsymbol{w}}^{U_{\perp}}$ & $\hat{v}_{\boldsymbol{p}, \boldsymbol{w}}^{U_{\top}}$ \\
\hline$\{1\}$ & $2 / 3$ & 1 \\
$\{2\}$ & $1 / 6$ & 0.4 \\
$\{3\}$ & $1 / 6$ & 0.4 \\
$\{1,2\}$ & 1 & 1 \\
$\{1,3\}$ & 1 & 1 \\
$\{2,3\}$ & $1 / 3$ & 1 \\
$N$ & 1 & 1 \\
\hline
\end{tabular}

Now, by expression (4) we get

$$
S_{\boldsymbol{p}, \boldsymbol{w}}^{U_{\perp}}(\boldsymbol{x})=\hat{v}_{\boldsymbol{p}, \boldsymbol{w}}^{U_{\perp}}(\{1\}) \cdot 2+\hat{v}_{\boldsymbol{p}, \boldsymbol{w}}^{U_{\perp}}(\{1,2\}) \cdot 2+\hat{v}_{\boldsymbol{p}, \boldsymbol{w}}^{U_{\perp}}(N) \cdot 6=28 / 3 .
$$

Therefore, given any semiuninorm $U \in \widetilde{\mathcal{U}}^{1 / n}$, by the second item of Proposition 1 we get $S_{\boldsymbol{p}, \boldsymbol{w}}^{U}(\boldsymbol{x}) \geq S_{\boldsymbol{p}, \boldsymbol{w}}^{U_{\perp}}(\boldsymbol{x})>\max \left(M_{\boldsymbol{p}}(\boldsymbol{x}), O_{\boldsymbol{w}}(\boldsymbol{x})\right)$.

Nevertheless, in the case of SUOWA operators we can establish conditions under which the property is satisfied for some specific semiuninorms.

Proposition 17. Let $\boldsymbol{p}$ and $\boldsymbol{w}$ be two weighting vectors such that $\sum_{i=1}^{j} w_{i} \leq$ $j / n$ for all $j \in N$ and $\min _{i \in N} p_{i}+\min _{i \in N} w_{i} \geq 1 / n$. Given $\boldsymbol{x} \in \mathbb{R}^{n}$, if $\min \left(M_{\boldsymbol{p}}(\boldsymbol{x}), O_{\boldsymbol{w}}(\boldsymbol{x})\right) \leq \overline{\boldsymbol{x}} \leq \max \left(M_{\boldsymbol{p}}(\boldsymbol{x}), O_{\boldsymbol{w}}(\boldsymbol{x})\right)$ then $\min \left(M_{\boldsymbol{p}}(\boldsymbol{x}), O_{\boldsymbol{w}}(\boldsymbol{x})\right) \leq$ $S_{\boldsymbol{p}, \boldsymbol{w}}^{U_{T_{\mathbf{L}}}}(\boldsymbol{x}) \leq \max \left(M_{\boldsymbol{p}}(\boldsymbol{x}), O_{\boldsymbol{w}}(\boldsymbol{x})\right)$.

Proof. Let $\boldsymbol{x} \in \mathbb{R}^{n}$ such that $\min \left(M_{\boldsymbol{p}}(\boldsymbol{x}), O_{\boldsymbol{w}}(\boldsymbol{x})\right) \leq \overline{\boldsymbol{x}} \leq \max \left(M_{\boldsymbol{p}}(\boldsymbol{x}), O_{\boldsymbol{w}}(\boldsymbol{x})\right)$. Suppose that $M_{\boldsymbol{p}}(\boldsymbol{x}) \leq \overline{\boldsymbol{x}} \leq O_{\boldsymbol{w}}(\boldsymbol{x})$ (the case $O_{\boldsymbol{w}}(\boldsymbol{x}) \leq \overline{\boldsymbol{x}} \leq M_{\boldsymbol{p}}(\boldsymbol{x})$ can be treated similarly). Then we have $M_{p}(\boldsymbol{x}) \leq M_{p}(\boldsymbol{x})+O_{\boldsymbol{w}}(\boldsymbol{x})-\overline{\boldsymbol{x}} \leq O_{\boldsymbol{w}}(\boldsymbol{x})$. Since by the third item of Proposition 9 we know that $S_{\boldsymbol{p}, \boldsymbol{w}}^{U_{\mathrm{L}}}(\boldsymbol{x})=M_{\boldsymbol{p}}(\boldsymbol{x})+O_{\boldsymbol{w}}(\boldsymbol{x})-\overline{\boldsymbol{x}}$, we conclude that $M_{\boldsymbol{p}}(\boldsymbol{x}) \leq S_{\boldsymbol{p}, \boldsymbol{w}}^{U_{T_{\mathbf{L}}}}(\boldsymbol{x}) \leq O_{\boldsymbol{w}}(\boldsymbol{x})$.

Proposition 18. Let $\boldsymbol{p}$ and $\boldsymbol{w}$ be two weighting vectors, and $U \in \mathcal{U}_{\mathrm{i}}^{1 / n}$. Then:

1. If $\mu_{\boldsymbol{p}} \leq \mu_{|\boldsymbol{w}|}$, then $M_{\boldsymbol{p}} \leq S_{\boldsymbol{p}, \boldsymbol{w}}^{U} \leq O_{\boldsymbol{w}}$.

2. If $\mu_{|\boldsymbol{w}|} \leq \mu_{\boldsymbol{p}}$, then $O_{\boldsymbol{w}} \leq S_{\boldsymbol{p}, \boldsymbol{w}}^{U} \leq M_{\boldsymbol{p}}$.

Proof. We will only consider the first statement (the proof of the second assertion is similar). By the first item of Proposition 8, we get $\mu_{\boldsymbol{p}} \leq \hat{v}_{\boldsymbol{p}, \boldsymbol{w}}^{U} \leq \mu_{|\boldsymbol{w}|}$. And now, by Remark 3, we have $M_{\boldsymbol{p}} \leq S_{\boldsymbol{p}, \boldsymbol{w}}^{U} \leq O_{\boldsymbol{w}}$. 
Notice that, although the assumptions of Proposition 18 are satisfied, WOWA operators still do not meet the property.

Example 4. Consider $\boldsymbol{p}=(0.3,0.3,0.2,0.1,0.1), \boldsymbol{w}=(0.4,0.3,0.1,0.1,0.1)$, and $\boldsymbol{x}=(10,10,8,6,6)$. It is easy to check that $\mu_{\boldsymbol{p}} \leq \mu_{|\boldsymbol{w}|}$ and that $M_{\boldsymbol{p}}(\boldsymbol{x})=8.8$ and $O_{\boldsymbol{w}}(\boldsymbol{x})=9$. Now, if $Q$ is a quantifier generating the weighting vector $\boldsymbol{w}$, then $Q(0)=0, Q(0.2)=0.4, Q(0.4)=0.7, Q(0.6)=0.8, Q(0.8)=0.9$, and $Q(1)=1$. According to expression (3), we get

$$
W_{\boldsymbol{p}, \boldsymbol{w}}^{Q}(\boldsymbol{x})=Q(0.3) \cdot 0+Q(0.6) \cdot 2+Q(0.8) \cdot 2+Q(0.9) \cdot 0+Q(1) \cdot 6=9.4,
$$

and, consequently, $W_{\boldsymbol{p}, \boldsymbol{w}}^{Q}(\boldsymbol{x})>\max \left(M_{\boldsymbol{p}}(\boldsymbol{x}), O_{\boldsymbol{w}}(\boldsymbol{x})\right)$.

Another interesting question is the following: given $\boldsymbol{y}, \boldsymbol{z} \in \mathbb{R}^{n}$, such that $M_{\boldsymbol{p}}(\boldsymbol{y}) \leq M_{\boldsymbol{p}}(\boldsymbol{z})$ and $O_{\boldsymbol{w}}(\boldsymbol{y}) \leq O_{\boldsymbol{w}}(\boldsymbol{z})$, under which conditions is it possible to guarantee that $W_{\boldsymbol{p}, \boldsymbol{w}}^{Q}(\boldsymbol{y}) \leq W_{\boldsymbol{p}, \boldsymbol{w}}^{Q}(\boldsymbol{z})\left(\right.$ or $\left.S_{\boldsymbol{p}, \boldsymbol{w}}^{U}(\boldsymbol{y}) \leq S_{\boldsymbol{p}, \boldsymbol{w}}^{U}(\boldsymbol{z})\right)$ ? As we can see in the following example, this property is not generally satisfied.

Example 5. Consider the weighting vectors of Example 3, $\boldsymbol{p}=(2 / 3,1 / 6,1 / 6)$ and $\boldsymbol{w}=(0.4,0.6,0)$, and $\boldsymbol{y}=(10,7,3), \boldsymbol{z}=(9,8,7)$. It is easy to check that $M_{\boldsymbol{p}}(\boldsymbol{y})=50 / 6<51 / 6=M_{\boldsymbol{p}}(\boldsymbol{z})$ and that $O_{\boldsymbol{w}}(\boldsymbol{y})=8.2<8.4=O_{\boldsymbol{w}}(\boldsymbol{z})$. In the case of WOWA operators, and according to expression (3), given any quantifier $Q$ generating the weighting vector $\boldsymbol{w}$ we have

$$
\begin{aligned}
& W_{\boldsymbol{p}, \boldsymbol{w}}^{Q}(\boldsymbol{y})=Q(2 / 3) \cdot 3+Q(5 / 6) \cdot 4+Q(1) \cdot 3=10 \\
& W_{\boldsymbol{p}, \boldsymbol{w}}^{Q}(\boldsymbol{z})=Q(2 / 3) \cdot 1+Q(5 / 6) \cdot 1+Q(1) \cdot 7=9,
\end{aligned}
$$

and, consequently, $W_{\boldsymbol{p}, \boldsymbol{w}}^{Q}(\boldsymbol{y})>W_{\boldsymbol{p}, \boldsymbol{w}}^{Q}(\boldsymbol{z})$. In the case of SUOWA operators, consider a semiuninorm $U \in \widetilde{\mathcal{U}}^{1 / n}$. Then

$$
\begin{aligned}
S_{\boldsymbol{p}, \boldsymbol{w}}^{U}(\boldsymbol{y})= & \hat{v}_{\boldsymbol{p}, \boldsymbol{w}}^{U}(\{1\}) \cdot 10+\left(\hat{v}_{\boldsymbol{p}, \boldsymbol{w}}^{U}(\{1,2\})-\hat{v}_{\boldsymbol{p}, \boldsymbol{w}}^{U}(\{1\})\right) \cdot 7 \\
& +\left(\hat{v}_{\boldsymbol{p}, \boldsymbol{w}}^{U}(N)-\hat{v}_{\boldsymbol{p}, \boldsymbol{w}}^{U}(\{1,2\})\right) \cdot 3 .
\end{aligned}
$$

Since $U_{\perp} \leq U \leq U_{\top}$, we get $\hat{v}_{\boldsymbol{p}, \boldsymbol{w}}^{U_{\perp}} \leq \hat{v}_{\boldsymbol{p}, \boldsymbol{w}}^{U} \leq \hat{v}_{\boldsymbol{p}, \boldsymbol{w}}^{U_{\top}}$ (see the proof of Proposition 8 in Llamazares $\left.^{8}\right)$. According to the values of Table 3 we have $2 / 3 \leq \hat{v}_{\boldsymbol{p}, \boldsymbol{w}}^{U}(\{1\}) \leq 1$ and $\hat{v}_{\boldsymbol{p}, \boldsymbol{w}}^{U}(\{1,2\})=\hat{v}_{\boldsymbol{p}, \boldsymbol{w}}^{U}(N)=1$. Therefore,

$$
S_{\boldsymbol{p}, \boldsymbol{w}}^{U}(\boldsymbol{y})=10 \alpha+7(1-\alpha)=7+3 \alpha,
$$

where $\alpha=\hat{v}_{\boldsymbol{p}, \boldsymbol{w}}^{U}(\{1\})$. Analogously,

$$
S_{\boldsymbol{p}, \boldsymbol{w}}^{U}(\boldsymbol{z})=9 \alpha+8(1-\alpha)=8+\alpha .
$$

Now we compare $S_{\boldsymbol{p}, \boldsymbol{w}}^{U}(\boldsymbol{y})$ and $S_{\boldsymbol{p}, \boldsymbol{w}}^{U}(\boldsymbol{z})$ :

$$
S_{\boldsymbol{p}, \boldsymbol{w}}^{U}(\boldsymbol{y})>S_{\boldsymbol{p}, \boldsymbol{w}}^{U}(\boldsymbol{z}) \Leftrightarrow 7+3 \alpha>8+\alpha \Leftrightarrow \alpha>0.5,
$$

which is true because $\alpha>2 / 3$. 
As we can see in the following propositions, in the case of SUOWA operators we can establish conditions under which the property is satisfied for some specific semi-uninorms.

Proposition 19. Let $\boldsymbol{p}$ and $\boldsymbol{w}$ be two weighting vectors such that $\sum_{i=1}^{j} w_{i} \leq j / n$ for all $j \in N$ and $\min _{i \in N} p_{i}+\min _{i \in N} w_{i} \geq 1 / n$. Given $\boldsymbol{y}, \boldsymbol{z} \in \mathbb{R}^{n}$, if $M_{\boldsymbol{p}}(\boldsymbol{y}) \leq$ $M_{\boldsymbol{p}}(\boldsymbol{z}), O_{\boldsymbol{w}}(\boldsymbol{y}) \leq O_{\boldsymbol{w}}(\boldsymbol{z})$ and $\overline{\boldsymbol{y}} \geq \overline{\boldsymbol{z}}$, then $S_{\boldsymbol{p}, \boldsymbol{w}}^{U_{T_{\mathbf{L}}}}(\boldsymbol{y}) \leq S_{\boldsymbol{p}, \boldsymbol{w}}^{U_{T_{\mathbf{L}}}}(\boldsymbol{z})$.

Proof. It is obvious by the third item of Proposition 9.

Proposition 20. Let $\boldsymbol{p}$ and $\boldsymbol{w}$ be two weighting vectors such that $\mu_{\boldsymbol{p}} \leq \mu_{|\boldsymbol{w}|}$, or $\mu_{|\boldsymbol{w}|} \leq \mu_{\boldsymbol{p}}$, and let $\boldsymbol{y}, \boldsymbol{z} \in \mathbb{R}^{n}$ with $\max \left(M_{\boldsymbol{p}}(\boldsymbol{y}), O_{\boldsymbol{w}}(\boldsymbol{y})\right) \leq \min \left(M_{\boldsymbol{p}}(\boldsymbol{z}), O_{\boldsymbol{w}}(\boldsymbol{z})\right)$. If $U \in \mathcal{U}_{\mathrm{i}}^{1 / n}$, then $S_{\boldsymbol{p}, \boldsymbol{w}}^{U}(\boldsymbol{y}) \leq S_{\boldsymbol{p}, \boldsymbol{w}}^{U}(\boldsymbol{z})$.

Proof. Suppose that $\mu_{p} \leq \mu_{|\boldsymbol{w}|}$ (the proof when the other condition is satisfied is similar). By Proposition 18, we get $S_{\boldsymbol{p}, \boldsymbol{w}}^{U}(\boldsymbol{y}) \leq O_{\boldsymbol{w}}(\boldsymbol{y}) \leq M_{\boldsymbol{p}}(\boldsymbol{z}) \leq S_{\boldsymbol{p}, \boldsymbol{w}}^{U}(\boldsymbol{z})$.

It is worthy of note that, although the assumptions of Proposition 20 are satisfied, WOWA operators do not fulfill the property.

Example 6. Consider again the weighting vectors of Example 4, where $\boldsymbol{p}=$ $(0.3,0.3,0.2,0.1,0.1)$ and $\boldsymbol{w}=(0.4,0.3,0.1,0.1,0.1)$, and $\boldsymbol{y}=(10,10,5,4,4), \boldsymbol{z}=$ $(9,9,8,7,7)$. It is easy to check that $\mu_{\boldsymbol{p}} \leq \mu_{|\boldsymbol{w}|}$, and that $\max \left(M_{\boldsymbol{p}}(\boldsymbol{y}), O_{\boldsymbol{w}}(\boldsymbol{y})\right)=$ $\max (7.8,8.3)=8.3<8.4=\min (8.4,8.5)=\min \left(M_{\boldsymbol{p}}(\boldsymbol{z}), O_{\boldsymbol{w}}(\boldsymbol{z})\right)$. According to expression (3), if $Q$ is a quantifier generating the weighting vector $\boldsymbol{w}$, we have

$$
\begin{aligned}
& W_{\boldsymbol{p}, \boldsymbol{w}}^{Q}(\boldsymbol{y})=Q(0.3) \cdot 0+Q(0.6) \cdot 5+Q(0.8) \cdot 1+Q(0.9) \cdot 0+Q(1) \cdot 4=8.9, \\
& W_{\boldsymbol{p}, \boldsymbol{w}}^{Q}(\boldsymbol{z})=Q(0.3) \cdot 0+Q(0.6) \cdot 1+Q(0.8) \cdot 1+Q(0.9) \cdot 0+Q(1) \cdot 7=8.7,
\end{aligned}
$$

and, consequently, $W_{\boldsymbol{p}, \boldsymbol{w}}^{Q}(\boldsymbol{y})>W_{\boldsymbol{p}, \boldsymbol{w}}^{Q}(\boldsymbol{z})$.

\section{CONCLUDING REMARKS}

WOWA and SUOWA operators have been purpose-built for dealing with situations where two weighting vectors are necessary: One of them provides information about the reliability of each information source, whereas the other one allows to weight the values according to their ordering. Both are Choquet integrals with respect to normalized capacities; therefore, they have properties such as continuity, monotonicity, idempotency, compensativeness, and homogeneity of degree 1. To make a distinction between both families, it seems interesting to analyze their behavior from other points of view. In this paper, we have studied these operators for some simple cases of weighting vectors, the capacities from which they are building, the weights affecting the components of each vector, and the values they return. We can summarize the main results as follows:

1. In some cases, WOWA operators grant more importance to the weighting vector $\boldsymbol{p}$ than to the weighting vector $\boldsymbol{w}$. In turn, it seems that SUOWA operators have a more equitable behavior between the weighting vectors (on this, see also Proposition 11 and Example 3 in Llamazares ${ }^{12}$ ). 
2. In the case of idempotent semiuninorms, the capacity of the SUOWA operator is located between the capacities of the weighted mean and the OWA operator. This is not the case for WOWA operators.

3. For some specific semiuninorms, and assuming suitable conditions on the weighting vectors, it is possible to give explicitly the weights $s_{i}$ or to guarantee that they range between the weights $p_{[i]}$ and $w_{i}$.

4. For some specific semiuninorms, and assuming some additional conditions, the value returned by the SUOWA operator lies between the values returned by the weighted mean and the OWA operator.

Finally, notice that some results of the paper are obtained by imposing the conditions $\sum_{i=1}^{j} w_{i} \leq j / n, \sum_{i=1}^{j} w_{i}<j / n$, or $\sum_{i=1}^{j} w_{i}>j / n$, for all $j \in N$. It is worth of emphasizing that some useful weighting vectors satisfy the previous conditions. For instance, those ones with increasing weights satisfy the condition $\sum_{i=1}^{j} w_{i} \leq j / n$ while those ones with decreasing weights satisfy the condition $\sum_{i=1}^{j} w_{i} \geq j / n$.

Proposition 21 (Llamazares $^{12}$ ). Let $\boldsymbol{w}$ be a weighting vector.

1. If $w_{1} \leq w_{2} \leq \cdots \leq w_{n}$, then $\boldsymbol{w}=\boldsymbol{\eta}$ or $\sum_{i=1}^{j} w_{i}<j / n$ for all $j \in$ $\{1, \ldots, n-1\}$.

2. If $w_{1} \geq w_{2} \geq \cdots \geq w_{n}$, then $\boldsymbol{w}=\boldsymbol{\eta}$ or $\sum_{i=1}^{j} w_{i}>j / n$ for all $j \in$ $\{1, \ldots, n-1\}$.

\section{Acknowledgment}

The author is grateful to an anonymous referee for valuable suggestions and comments. The partial financial support from the Ministerio de Economía y Competitividad (Project ECO2012-32178) and the Junta de Castilla y León (Consejería de Educación, Project VA066U13) is gratefully acknowledged.

\section{References}

1. Yager RR. On ordered weighted averaging operators in multicriteria decision making. IEEE Trans Syst, Man, Cybern 1988;18:183-190.

2. Torra V. Weighted OWA operators for synthesis of information. In: Proceedings of the Fifth IEEE International Conference on Fuzzy Systems, vol. 2; 1996. pp 966-971.

3. Torra V. The weighted OWA operator. Int J Intell Syst 1997;12:153-166. 
4. Torra V, Godo L. Continuous WOWA Operators with Application to Defuzzification. In: Calvo T, Mayor G, Mesiar R, editors, Aggregation Operators: New Trends and Applications, Studies in Fuzziness and Soft Computing, vol. 97, 159-176. Heidelberg: Physica-Verlag, 2002.

5. Torra V, Narukawa Y. Modeling Decisions: Information Fusion and Aggregation Operators. Berlin: Springer, 2007.

6. Roy B. Double pondération pour calculer une moyenne: Pourquoi et comment? RAIRO - Oper Res 2007;41:125-139.

7. Yager RR, Alajlan N. A generalized framework for mean aggregation: Toward the modeling of cognitive aspects. Inform Fusion 2014;17:65-73.

8. Llamazares B. Constructing Choquet integral-based operators that generalize weighted means and OWA operators. Inform Fusion 2015;23:131-138.

9. Llamazares B. An analysis of some functions that generalizes weighted means and OWA operators. Int J Intell Syst 2013;28:380-393.

10. Liu HW. Semi-uninorms and implications on a complete lattice. Fuzzy Sets Syst 2012;191:72-82.

11. Yager RR, Rybalov A. Uninorm aggregation operators. Fuzzy Sets Syst 1996;80:111-120.

12. Llamazares B. SUOWA operators: Constructing semi-uninorms and analyzing specific cases. Fuzzy Sets Syst (in press). DOI: 10.1016/j.fss.2015.02.017.

13. Llamazares B. A study of SUOWA operators in two dimensions. Math Probl Eng 2015;2015:Article ID 271491, 12 pages.

14. Choquet G. Theory of capacities. Ann Inst Fourier 1953;5:131-295.

15. Grabisch M, Murofushi T, Sugeno M, editors. Fuzzy Measures and Integrals: Theory and Applications, Studies in Fuzziness and Soft Computing, vol. 40. Heidelberg: Physica-Verlag, 2000.

16. Grabisch M, Labreuche C. A decade of application of the Choquet and Sugeno integrals in multi-criteria decision aid. Ann Oper Res 2010;175:247286.

17. Murofushi T, Sugeno M. A theory of fuzzy measures. Representation, the Choquet integral and null sets. J Math Anal Appl 1991;159:532-549.

18. Sugeno M. Theory of Fuzzy Integrals and its Applications. PhD thesis, Tokyo Institute of Technology, 1974.

19. Maschler M, Peleg B. The structure of the kernel of a cooperative game. SIAM J Appl Math 1967;15:569-604. 
20. Maschler M, Peleg B, Shapley LS. The kernel and bargaining set for convex games. Int J Game Theory 1971;1:73-93.

21. Denneberg D. Non-Additive Measures and Integral. Dordrecht: Kluwer Academic Publisher, 1994.

22. Grabisch M, Marichal J, Mesiar R, Pap E. Aggregation Functions. Cambridge: Cambridge University Press, 2009.

23. Torra V. On some relationships between the WOWA operator and the Choquet integral. In: Proc. 7th Int. Conf. on Information Processing and Management of Uncertainty in Knowledge-Based Systems (IPMU'98); 1998. pp 818-824.

24. Wilkin T, Beliakov G. Weakly monotonic averaging functions. Int J Intell Syst 2015;30:144-169.

25. Emrouznejad A, Marra M. Ordered weighted averaging operators 1988-2014: A citation-based literature survey. Int J Intell Syst 2014;29:994-1014.

26. Fodor J, Marichal JL, Roubens M. Characterization of the ordered weighted averaging operators. IEEE Trans Fuzzy Syst 1995;3:236-240.

27. Grabisch M. Fuzzy integral in multicriteria decision making. Fuzzy Sets Syst 1995;69:279-298.

28. Grabisch M. On equivalence classes of fuzzy connectives - the case of fuzzy integrals. IEEE Trans Fuzzy Syst 1995;3:96-109.

29. Torra V, Godo L. Averaging continuous distributions with the WOWA operator. In: Proc. 2nd Eur. Workshop on Fuzzy Decision Analysis and Neural Networks for Management, Planning and Optimization (EFDAN'97); 1997. pp 10-19.

30. Yager RR. Families of OWA operators. Fuzzy Sets Syst 1993;59:125-148.

31. Marichal JL. An axiomatic approach of the discrete Choquet integral as a tool to aggregate interacting criteria. IEEE Trans Fuzzy Syst 2000;8:800-807. 